\title{
Pan-Genome Analysis of Human Gastric Pathogen $H$. pylori: Comparative Genomics and Pathogenomics Approaches to Identify Regions Associated with Pathogenicity and Prediction of Potential Core Therapeutic Targets
}

\author{
Amjad Ali, ${ }^{1,2}$ Anam Naz, ${ }^{1}$ Siomar C Soares, ${ }^{2}$ Marriam Bakhtiar, ${ }^{2,3}$ Sandeep Tiwari, ${ }^{2}$ \\ Syed S Hassan, ${ }^{2}$ Fazal Hanan, ${ }^{4}$ Rommel Ramos, ${ }^{5}$ Ulisses Pereira, ${ }^{2,6}$ Debmalya Barh, ${ }^{7}$ \\ Henrique César Pereira Figueiredo, ${ }^{6}$ David W. Ussery, ${ }^{8}$ Anderson Miyoshi, ${ }^{2}$ \\ Artur Silva, ${ }^{5}$ and Vasco Azevedo ${ }^{2}$ \\ ${ }^{1}$ Atta-ur-Rahman School of Applied Biosciences (ASAB), National University of Sciences and Technology (NUST), \\ Islamabad 44000, Pakistan \\ ${ }^{2}$ Laboratory of Cellular and Molecular Genetics, Federal University of Minas Gerais (UFMG), 31907-270 Belo Horizonte, MG, Brazil \\ ${ }^{3}$ Department of Bioinformatics, Mohammad Ali Jinnah University (MAJU), Sehala Road, Islamabad 44000, Pakistan \\ ${ }^{4}$ KIMS, Khyber Medical University, Peshawar 25000, Pakistan \\ ${ }^{5}$ Federal University of Pará, 66075-110 Belém, PA, Brazil \\ ${ }^{6}$ Laboratory of Aquatic Animal Diseases (AQUAVET), Department of Preventive Veterinary Medicine, \\ Federal University of Minas Gerais, 31907-270 Belo Horizonte, MG, Brazil \\ ${ }^{7}$ Centre for Genomics and Applied Gene Technology, Institute of Integrative Omics and Applied Biotechnology (IIOAB), \\ Nonakuri, Purba Medinipur, West Bengal 721172, India \\ ${ }^{8}$ Centre for Biological Sequence Analysis (CBS), Technical University of Denmark, 2800 Kongens Lyngby, Denmark
}

Correspondence should be addressed to Vasco Azevedo; vasco@icb.ufmg.br

Received 3 February 2014; Revised 11 July 2014; Accepted 11 July 2014

Academic Editor: Angel Cataldi

Copyright (C) 2015 Amjad Ali et al. This is an open access article distributed under the Creative Commons Attribution License, which permits unrestricted use, distribution, and reproduction in any medium, provided the original work is properly cited.

\begin{abstract}
Helicobacter pylori is a human gastric pathogen implicated as the major cause of peptic ulcer and second leading cause of gastric cancer $(\sim 70 \%)$ around the world. Conversely, an increased resistance to antibiotics and hindrances in the development of vaccines against $H$. pylori are observed. Pan-genome analyses of the global representative $H$. pylori isolates consisting of 39 complete genomes are presented in this paper. Phylogenetic analyses have revealed close relationships among geographically diverse strains of $H$. pylori. The conservation among these genomes was further analyzed by pan-genome approach; the predicted conserved gene families $(1,193)$ constitute $\sim 77 \%$ of the average $H$. pylori genome and $45 \%$ of the global gene repertoire of the species. Reverse vaccinology strategies have been adopted to identify and narrow down the potential core-immunogenic candidates. Total of 28 nonhost homolog proteins were characterized as universal therapeutic targets against $H$. pylori based on their functional annotation and protein-protein interaction. Finally, pathogenomics and genome plasticity analysis revealed 3 highly conserved and 2 highly variable putative pathogenicity islands in all of the $H$. pylori genomes been analyzed.
\end{abstract}

\section{Background}

The genus Helicobacter includes bacterial species which colonizes the gastrointestinal tract (GIT) of human and other mammals. Both $H$. pylori and non-pylori species in the genus are involved in gastrointestinal diseases [1,2]. In humans, $H$. pylori causes diseases like gastritis and peptic ulcers, which can lead to the development of gastric cancer $(\sim 10 \%$ of cancer deaths around the globe). Therefore, the pathogen was enlisted as a class I carcinogen by WHO in 1994 [2, 3]. 
The infection rates remain as high as $90 \%$ in developing world, and in low socioeconomic regions like the ones located in Africa, East Asia, and Central America the diseases (peptic ulcer and gastric cancer) are highly prevalent. However, in the developed world and industrialized cities the prevalence is considerably lower, most probably due to efficient preventive and public health measures taken by some western countries [4].

Previous studies indicated that most of the $H$. pylori isolates could be classified either by sequence diversity or gene content. This is the main reason of getting certainly great differences in isolates of $H$. pylori strains from different continents. These genomic variations are indicative of genetic drift during geographic isolation, adaptation, and coevolution of the pathogen within different ethnic groups of human population $[5,6]$.

The mechanisms of $H$. pylori pathogenesis are relatively complex than other bacterial strains; however, some wellknown factors such as the type IV secretion system (T4SS) and the CagA oncoprotein have been demonstrated to play an important role in inflammation and carcinogenesis of GIT [7, 8]. Beside these, there are few other virulence factors which have been identified and discussed in the literature. These factors help in identifying the infectious agents and disease causing behavior of the said bacterial specie. H. pylori has a diverse genome and carries many plasticity zones which differ among strains. Thus, the identification of these and other novel regions in a particular strain would offer insights into the pathogenic diversity of $H$. pylori strains [9]. A detailed phylogenomics and comparative genomics study is required to understand the association among different isolates and disease symptoms.

To explore the conserved features and shared genomic contents, Salama et al., in 2000, attempted to determine the core set of genes in $15 \mathrm{H}$. pylori strains by microarray method and observed that 1,281 genes are shared by all the examined strains. However, the examined strains were mainly isolated from western countries $[10,11]$. For detailed view of the diversity and conservation in $H$. pylori strains, Gressmann et al., in 2005, determined the core genome of a larger set and global representative strains and suggested that $H$. pylori core genome consists of 1,111 genes [6].

These findings indicated that as the number of genome sequences increases, the actual or true core genome can be obtained; furthermore, the genomic diversity may also be predicted. Among the genetic repertoire of the H. pylori including genes for various functions, the core minimal set of genes is of particular interest. It is required for maintaining the basic cellular life and is indispensible for survival of the organism [12]. Calculating the conserved/core genome of diverse strains of $H$. pylori at larger scale would provide an overview of strain diversity and this data could be utilized for further use in diagnostics and therapeutics applications.

Furthermore, the identification and characterization of core essential genes, in other words the minimal essential genes set, in $H$. pylori species would be an interesting strategy both theoretically and experimentally to understand the basic requirements for cellular life. A practical example of the importance of minimal gene set characterization is in drug development. Drugs are usually designed against essential cellular processes and essential gene products of microbial cells as they are specific and provide promising new targets for such drugs [13].

Recently, we witnessed an increase in the number of complete genome sequences of $H$. pylori on public databases. With this, we intended to follow reverse vaccinology strategy to predict the potential core-immunogenic, virulence factors which can serve as putative vaccine candidates against $H$. pylori and, moreover, to utilize all those available complete genome sequences to get insights into the genomic diversity in $H$. pylori species and to compare the genomes. We believe that comparative analysis of this pathogen (H. pylori) genome sequences and their nonpathogenic relative will offer a unique opportunity for the identification of regions of genomes such as novel pathogen specific pathogenicity islands $[9,14,15]$.

\section{Material and Methods}

2.1. Data Collection and Management. The gastric pathogen $H$. pylori have multiple complete genomes available on public databases for scientific exploitation. Here, we selected a total of 39 complete $H$. pylori genomes (available at the time of analysis). The draft or incomplete genomes were not included for instance, to have uniformity in the analyses. GenBank (gbk) files were obtained from NCBI genome browser (http://www.ncbi.nlm.nih.gov/genome/browse/). As a starting point, DNA sequences in a fast format were extracted from all the GenBank files (chromosome and plasmid sequences were merged, as they were required) and then subjected to program RNAmmer [16] for prediction of full length $16 \mathrm{~S}$ rRNA gene sequences. The program Prodigal, a gene finding algorithm, was also used to predict open reading frames (ORFs) in all genomes [17]. We, however, have seen slight variations in the number of genes/ORFs in our predicted ones and those available publicly (GenBank). To avoid inconsistencies and to get uniformity in the resultant data, we used a single gene finding program. Table 1 demonstrates the basic genome information, that is, size in base pairs, number of predicted proteins, chromosome/plasmid accession numbers, and percent AT content.

2.2. Phylogenetic Analysis. In order to better understand the evolutionary relationships and genomic variations at genus level, all 39 sequences genomes of $H$. pylori species were analyzed for 16S rRNA genes using RNAmmer [16]. Multiple sequence alignment of all obtained 16S rRNA genes was aligned using ClustalW [18]. Sequences generated with score $>1700$ are usually considered to be more reliable and necessary for phylogenetic analysis $[16,19]$. Phylogenetic tree was generated following Neighbor Joining algorithm [20] by using MEGA6 [21].

2.3. Proteome Comparisons (Pairwise Alignment). All the selected $H$. pylori genomes were translated into their proteomes. BLASTp comparisons were carried out for all the proteins present in one genome against all the proteins in the other genomes in the study $[19,22]$. The BLAST parameters were set as follows: BLAST cutoff $e$-value was $1 e-5$; 
TABLE 1: List of genomes analyzed in this study. Genomic feature, statistics, accession numbers (chromosome + plasmid) and pangenomic data generated by analyzing 39 Helicobacter pylori strains. The number of predicted proteins, clusters, new genes (unique), and core and pan genome is estimated.

\begin{tabular}{|c|c|c|c|c|c|c|c|c|c|}
\hline \multirow[b]{2}{*}{ Organisms } & \multicolumn{4}{|c|}{ Genomic features and statistics } & \multicolumn{5}{|c|}{ Pan-genomics and comparative genomics } \\
\hline & Size (bp) & Proteins & Chromosome & Plasmid & $\%$ AT & $\begin{array}{l}\text { New } \\
\text { genes }\end{array}$ & $\begin{array}{c}\text { New } \\
\text { families }\end{array}$ & $\begin{array}{c}\text { Pan- } \\
\text { genome }\end{array}$ & $\begin{array}{c}\text { Core } \\
\text { genome }\end{array}$ \\
\hline Helicobacter pylori 2017 & 1548238 & 1503 & NC_017374.1 & & 60.698 & 1503 & 1491 & 1491 & 1491 \\
\hline Helicobacter pylori 2018 & 1562832 & 1508 & NC_017381.1 & & 60.706 & 29 & 29 & 1520 & 1464 \\
\hline Helicobacter pylori 26695 & 1667867 & 1579 & NC_000915.1 & & 61.124 & 207 & 189 & 1702 & 1323 \\
\hline Helicobacter pylori 35A & 1566655 & 1500 & NC_017360.1 & & 61.132 & 106 & 103 & 1800 & 1291 \\
\hline Helicobacter pylori 51 & 1589954 & 1515 & NC_017382.1 & & 61.230 & 80 & 79 & 1871 & 1278 \\
\hline Helicobacter pylori 52 & 1568826 & 1509 & NC_017354.1 & & 61.059 & 63 & 62 & 1926 & 1273 \\
\hline Helicobacter pylori 83 & 1617426 & 1559 & NC_017375.1 & & 61.277 & 87 & 84 & 2004 & 1268 \\
\hline Helicobacter pylori 908 & 1549666 & 1511 & NC_017357.1 & & 60.701 & 24 & 24 & 2023 & 1264 \\
\hline Helicobacter pylori B38 & 1576758 & 1502 & NC_012973.1 & & 60.840 & 61 & 52 & 2065 & 1237 \\
\hline Helicobacter pylori B8 6296 & 1680029 & 1579 & NC_014256.1 & NC_014257.1 & 61.215 & 51 & 50 & 2097 & 1235 \\
\hline Helicobacter pylori Cuz20 & 1635449 & 1536 & NC_017358.1 & & 61.136 & 73 & 72 & 2153 & 1227 \\
\hline Helicobacter pylori ELS37 & 1669876 & 1564 & NC_017063.1 & NC_017064.1 & 61.123 & 57 & 55 & 2194 & 1224 \\
\hline Helicobacter pylori F16 & 1575399 & 1508 & NC_017368.1 & & 61.115 & 28 & 26 & 2205 & 1225 \\
\hline Helicobacter pylori F30 & 1579693 & 1498 & NC_017365.1 & NC_017369.1 & 61.197 & 19 & 19 & 2210 & 1225 \\
\hline Helicobacter pylori F32 & 1581461 & 1506 & NC_017366.1 & NC_017370.1 & 61.143 & 21 & 21 & 2218 & 1223 \\
\hline Helicobacter pylori F57 & 1609006 & 1530 & NC_017367.1 & & 61.272 & 16 & 16 & 2226 & 1221 \\
\hline Helicobacter pylori G27 & 1663013 & 1577 & NC_011333.1 & NC_011334.1 & 61.130 & 51 & 51 & 2266 & 1221 \\
\hline Helicobacter pylori Gambia 9424 & 1712468 & 1589 & NC_017371.1 & NC_017364.1 & 60.876 & 28 & 28 & 2286 & 1220 \\
\hline Helicobacter pylori HPAG1 & 1605736 & 1508 & NC_008086.1 & NC_008087.1 & 60.933 & 32 & 32 & 2310 & 1220 \\
\hline Helicobacter pylori HUP-B14 & 1607584 & 1507 & NC_017733.1 & NC_017734.1 & 60.957 & 37 & 35 & 2339 & 1219 \\
\hline Helicobacter pylori India7 & 1675918 & 1572 & NC_017372.1 & & 61.102 & 33 & 32 & 2362 & 1217 \\
\hline Helicobacter pylori $\mathrm{J99}$ & 1643831 & 1505 & NC_000921.1 & & 60.811 & 18 & 18 & 2373 & 1216 \\
\hline Helicobacter pylori Lithuania75 & 1640673 & 1555 & NC_017362.1 & NC_017363.1 & 61.134 & 42 & 42 & 2401 & 1212 \\
\hline Helicobacter pylori P12 & 1684038 & 1587 & NC_011498.1 & NC_011499.1 & 61.213 & 22 & 22 & 2411 & 1206 \\
\hline Helicobacter pylori PeCan18 & 1660685 & 1543 & NC_017742.1 & & 60.981 & 17 & 17 & 2415 & 1208 \\
\hline Helicobacter pylori PeCan 4 & 1638269 & 1532 & NC_014555.1 & NC_014556.1 & 61.089 & 27 & 27 & 2435 & 1208 \\
\hline Helicobacter pylori Puno120 & 1637762 & 1532 & NC_017378.1 & NC_017377.1 & 61.095 & 26 & 26 & 2452 & 1206 \\
\hline Helicobacter pylori Puno135 & 1646139 & 1539 & NC_017379.1 & & 61.176 & 14 & 14 & 2463 & 1205 \\
\hline Helicobacter pylori SAfrica7 & 1679829 & 1573 & NC_017361.1 & NC_017373.1 & 61.575 & 48 & 47 & 2498 & 1206 \\
\hline Helicobacter pylori Sahul64 & 1644275 & 1579 & ALWV01 & & 61.240 & 32 & 30 & 2513 & 1205 \\
\hline Helicobacter pylori Sat464 & 1567570 & 1464 & NC_017359.1 & NC_017356.1 & 60.908 & 15 & 15 & 2521 & 1205 \\
\hline Helicobacter pylori Shill2 & 1663456 & 1561 & NC_017741.1 & & 61.227 & 10 & 10 & 2519 & 1205 \\
\hline Helicobacter pylori Shi169 & 1616909 & 1516 & NC_017740.1 & & 61.136 & 6 & 6 & 2521 & 1205 \\
\hline Helicobacter pylori Shi417 & 1665719 & 1545 & NC_017739.1 & & 61.229 & 14 & 14 & 2530 & 1204 \\
\hline Helicobacter pylori Shi470 & 1608548 & 1518 & NC_010698.2 & & 61.229 & 7 & 7 & 2530 & 1203 \\
\hline Helicobacter pylori SJM180 & 1658051 & 1524 & NC_014560.1 & & 61.100 & 15 & 15 & 2540 & 1203 \\
\hline Helicobacter pylori SNT49 & 1610830 & 1514 & NC_017376.1 & NC_017380.1 & 61.004 & 12 & 12 & 2540 & 1202 \\
\hline Helicobacter pylori v225d & 1595604 & 1510 & NC_017355.1 & NC_017383.1 & 61.055 & 15 & 15 & 2549 & 1199 \\
\hline Helicobacter pylori XZ274 & 1656544 & 1701 & NC_017926.1 & NC_017919.1 & 61.427 & 89 & 89 & 2614 & 1193 \\
\hline
\end{tabular}


homologs were selected at $e$-value of $1 e-8$; identity homolog was 0.95 ; the blast minimum score was 30 ; and minimum identity was 90. Reciprocal best blast hits were selected for generation of blast matrix which is shown in Figure 2. Each cell in the blast matrix demonstrates genes/proteins ( $x$-axis) reciprocal hits with respect to the genome listed to the right ( $y$-axis). Each corresponding rectangular box (between any two genomes) in the matrix represents the number of reciprocal hits (shared proteins) and the total number of proteins. For example, two genomes, G1 and G2, will lead to $(\mathrm{G} 1 / \mathrm{G} 2) * 100=\mathrm{X} \%$ of the proteins/genes in $\mathrm{G} 1$ had reciprocal hits, while $(\mathrm{G} 2 / \mathrm{G} 1) * 100=\mathrm{X} \%$ of the proteins/genes in G2 had reciprocal hits. The diagonal row of boxes indicates the internal homologies against itself (genome). The colored matrix is generated for all the 39 genomes/proteomes with the scale given, indicating the relative homology between corresponding genome/proteome.

2.4. Pan-Genome Analysis and Characterization of Core Genome. The H. pylori conserved core families (CGFs) and pan-genome families (PGFs) were estimated followed by previously established method $[19,22]$. CGFs and PGFs were estimated by employing single-linkage clustering on top of BLASTp alignments, with the notion that any two genes in the data set are considered to belong to the same gene family and should be considered as "conserved" if their amino acid sequences are at least $50 \%$ identical over at least $50 \%$ of the length of the longest gene $[22,23]$. The strategy may also be termed as 50/50. Doing so, multiple genes may belong to single gene family and the number of gene families would be lower than the actual number of genes in a genome. Those genes which do not fit to the criterion would constitute individual genes families. Gene families with at least one gene in common were gathered into the core genome. The rest, either unmatched or not qualifying according to the criterion, constitute the species pan-genome [24-26].

2.5. Essential Gene Families and Nonhost Homologs Prediction. The $H$. pylori predicted core genome was then aligned with the Database for Essential Genes, DEG (http://tubic.tju.edu.cn/deg/), for estimation of essential core gene families, EGFs [12]. DEG contains essential genes data from 32 bacteria, including E. coli, B. subtilis, H. pylori, S. pneumoniae, $M$. genitalium, and $H$. influenzae. The BLAST comparison settings for selecting the essential genes/proteins were followed as previously described [27]: expected value $(e-$ value) cutoff of $10^{-10}$; percentage of identity $\geq 35 \%$ between query and hits; and minimum bit score of 100. In the case of $H$. pylori, the host (human) genome/proteome was downloaded from NCBI (taxid: 9606) and BLASTp analysis were performed. In BLASTp comparison parameters, the percentages of identity and $e$-value were kept $<35 \%$ and 0.005 , respectively. Proteins without hits below the $e$-value inclusion threshold were collected as nonhost bacterial proteins and called nonhost essential gene families (nHEGFs).

2.6. Screening Core Exoproteome. For prediction of the potential immunogenic and vaccine candidate genes and proteins, the predicted nHEGFs (283) were analyzed for their surface localization and signal peptide detection using CELLO (subCELlular LOcalization predictive system; http://cello.life.nctu.edu.tw/) and PSORTb (http://www.psort .org/psortb/). Exoproteome and secretome always remain a good source for therapeutic targets. Moreover, for the prediction of potential vaccine targets and analyses, proteins were analyzed for their transmembrane helices, MHC class I and II binding epitopes, nonhost conservation, and pathogen specific proteins.

2.7. Prioritizing Vaccine Targets. Proteins falling in nHEGFs category were then analyzed for their potential as therapeutic targets. Proteins having $<110 \mathrm{KDa}$ are considered to be good therapeutic targets. Proteins labeled as surface or secreting proteins predicted from CELLO and PSORTb in previous step were subjected to ExPASy PI/MW tool [28] which calculated their molecular weight.

2.8. Functional Annotation of Targets. Predicted surface and secreting proteins were then analyzed for functional annotation using Blast2Go [29] which is a workstation for functional annotation of nucleotide/protein sequences; the program not only provide annotated results but also give remote access to user which help in analyzing data with different graphical and statistical functions.

2.9. Protein-Protein Interactions (PPIs) of Prioritized Targets. Proteome-scale interaction analysis of prioritized proteins was performed using Search Tool for the Retrieval of Interacting Genes (STRING) (http://www.string-db.org) [30]. STRING being a comprehensive database and user friendly tool provides a good opportunity of protein-protein interactions along with their available information regarding functional categorization and domain evaluation, and so forth.

2.10. Epitope Mapping. To predict best vaccine targets prioritized proteins with their full length sequence were subjected to BCPred analysis [31] to predict B-cell epitopes. Cutoff score for 20-mer epitope prediction was maintained as 0.8 and epitopes having $>0.8$ value were then subjected to VaxiJen [32] to check antigenicity (threshold $=0.4$, ACC output).

2.11. Pathogenomic Analysis and Prediction of Pathogenicity Islands. In order to identify pathogenicity islands (PAIs) in H. pylori species, we have performed an analysis with PIPS (Pathogenicity Island Prediction Software) [33]. PIPS identifies PAIs according to their main feature: genomic signature deviations, that is, $\mathrm{G}+\mathrm{C}$ content and codon usage deviations; presence of virulence factors, transposase genes and flanking tRNAs genes; and absence in nonpathogenic organism from the same genus or related species. The analyses were performed on $H$. pylori strains 26695, Cuz-20, J99, PeCan4, and SouthAfrica7, which are representative of Europe, East Asia, West Africa, South America, and South Africa, respectively. Additionally, Wolinella succinogenes DSM 1740 was chosen as nonpathogenic closely related species for PIPS analysis requirement [34]. After prediction step, the sizes of all PAIs 
in all $5 \mathrm{H}$. pylori genomes used in this step were manually inspected through graphical genome comparisons in ACT: the Artemis Comparison tool [35]. Finally, we obtained reference PAIs from the genomes which can be used as representative islands among all. The reference PAIs were then compared with all 39 genomes of $H$. pylori included in this study using the proteome comparison data and plotted a heatmap for easy visualization of percentage identity.

\section{Results and Discussion}

3.1. H. pylori Genome Statistics and Features. H. pylori is a Gram-negative microaerophilic bacterium which colonizes the human stomach, a pathogen belonging to epsilonbacteria. $[1,36,37]$. $H$. pylori species has a total of 39 complete genomes sequences on NCBI GenBank (at the time of analyses). The majority of them are in Refseq. Of the $39 \mathrm{H}$. pylori complete genomes, about half of them (17) carry plasmid DNA (one or more) (Table 1). The total number of proteins in $39 \mathrm{H}$. pylori genomes was calculated as 59,958 , while an average $H$. pylori genome was found to contain 1,537 protein coding genes (CDS). The genome sequencing of this important pathogen started with $H$. pylori 26695 , which was obtained in 1997. It has been observed that it contains a circular chromosome of $1,667,867$ base pairs with an average GC content of 39\% [36]. Later on, while other genomes were sequenced it became prominent that $H$. pylori genomes have uniform (lower) GC contents. According to GenBank data, the \%GC does not have considerable variations and range from 38 to $39 \%$ (http://www.ncbi.nlm.nih.gov/genome/browse/). During the analyses of $H$. pylori genomes, an average $61 \%$ of AT content was observed. The closely related strain $\mathrm{J} 99$ isolated from an American patient having duodenal ulcer; when compared to H. pylori, 26695, a total of 1,406 genes were found common amongst them. The J99 was observed to have 86 unique genes; however, both strains share the important cag pathogenicity island, which codes for the type IV secretion system, facilitating the transport of CagA cytotoxin to gastric epithelial cells $[3,38]$. Later, the strain HPAG1 (isolated from an elderly women in Sweden) sequenced in 2006 [39] was compared to the previous two strains (J99 and 26 695) and it has a smaller genome size and shared the CagA and vacA (virulent allele). HPAG1 was found to contain 43 strain specific genes. Another strain, G27, which is similar in size (1652983 bp) to the previous strains (26 695, J99, and HPAG1), contains a plasmid (11 genes) and 58 specific genes. However, the cag island is reported to be disrupted by a transposon in G27 [3, 8]. The strain Shi470 has a genome relatively larger in size $(\sim 1.6 \mathrm{Mbp})$ than the previous strains. The recently sequenced genome H. pylori Sahul64 (ALWV01) isolated from an Australian Aboriginals also lies in the range and has a genome 1.64 Mbp consisting of 1579 predicted genes [40]. However, there is a consensus in the scientific community that there is an increase in $H$. pylori genomic variability and the phenomena of mutations and recombination are continuously occurring. The analyzed genomes in this study and their genome statistics, shared genes/proteins, and genes associated to particular strains were predicted and are shown in Table 1. For example, the highest number of strain specific genes families (189 GFs-207 genes) was observed in the $H$. pylori strain 26695 while the lowest (6 GFs) was found in $H$. pylori Shil69.

3.2. Phylogenetic Analysis of Ribosomal RNA. H. pylori is a well-recognized human gastric pathogen, being colonizing the earliest human populations and it is believed to have coevolved and comigrated with its hosts across the world [4]. A phylogenetic analysis has been done for $39 \mathrm{H}$. pylori species to understand pattern of evolution and distribution of the organism. The phylogenetic tree based on 16S rRNA was generated from the resultant data (extracted from genomes) using MEGA6 [21]. Neighbor Joining method was employed to construct the tree [41]. After generating possible pairwise and multiple alignments MEGA6 illustrates a phylogenetic tree representing the optimal tree with the sum of branch length $=0.04530419$. The tree is drawn to scale, with branch lengths in the same units as those of the evolutionary distances used to infer the phylogenetic tree (Figure 1). The evolutionary distances were computed using the Maximum Composite Likelihood method [42] and are in the units of the number of base substitutions per site. The analysis involved 39 nucleotide sequences. Codon positions included were $1 s t+2 n d+3 r d+$ noncoding. All positions containing gaps and missing data were eliminated. There were a total of 1482 positions in the final dataset. A newly sequenced strain (Sahul64) isolated from Australian Aboriginals [40] has a draft genome available and incorporation of this genome in phylogenetic analysis shows maximum branch length in its clade representing the higher evolutionary time. The previous reports have revealed that the $H$. pylori species divides into two clusters: one with the species which colonize the stomach of mammals (so called gastric species) and the other with species that inhabit the intestine and biliary tracts (so called enterohepatic cluster) [5]. To better understand intraspecies relationships in $H$. pylori the amount and percentage of shared proteome are predicted.

3.3. Proteome Conservation in H. pylori Strains (Pairwise Alignment). We observed higher similarities in genomic contents of $H$. pylori strains in phylogenomic analysis; therefore, we decided to compare the whole predicted proteome (proteins) of all $H$. pylori strains to estimate the amount of proteins they share. The $H$. pylori genomes were pairwise compared (BLASTp). To estimate the amount of shared proteome between and among strains, a matrix is generated from the data of comparisons which is shown in Figure 2. Both number and percentage of shared proteome (proteins) are shown in corresponding box of the matrix. For example, we can see in the color matrix a maximum proteome conservation of $\sim 98 \%$ and a minimum of $\sim 81 \%$. The maximum similarity we observed is in H. pylori 2017 and H. pylori 2018 and vice versa. Inversely to this, one can see the minimum $\sim 81 \%$ proteome similarity between H. pylori 2018 and H. pylori Shi 470. Similarly, H. pylori 908 and $H$. pylori B8 share the same amount of proteins with $H$. pylori Shi 470 . These findings are interesting when compared with phylogenetic trees and reflect the consistent 


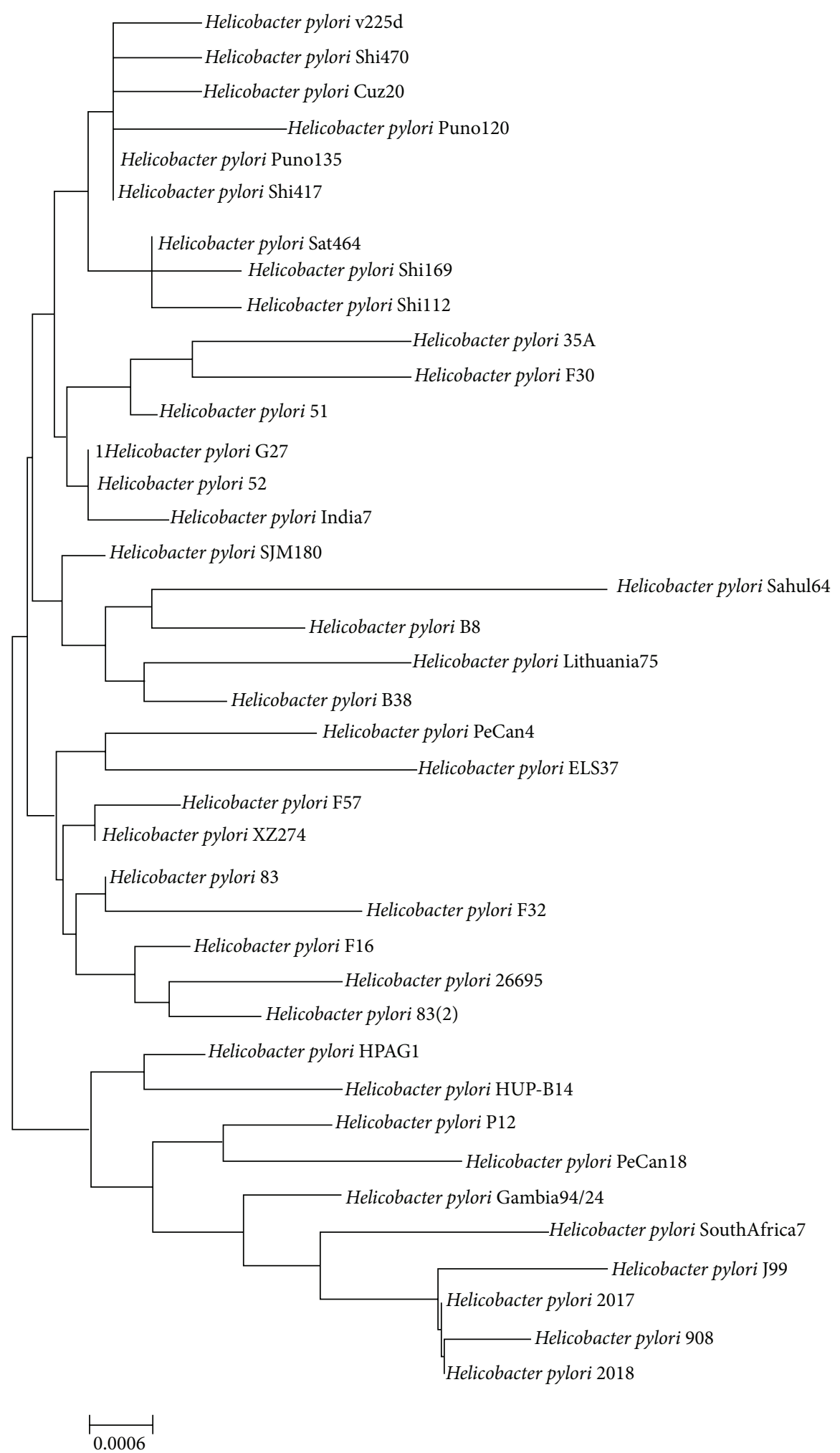

FIgURE 1: The evolutionary history inferred by Neighbor Joining method (NJ). MEGA6 was used for multiple sequence alignment and construction of phylogenetic tree of all $39 \mathrm{H}$. pylori strains. Branch lengths were computed using evolutionary distances generated by Maximum Composite Likelihood method. 


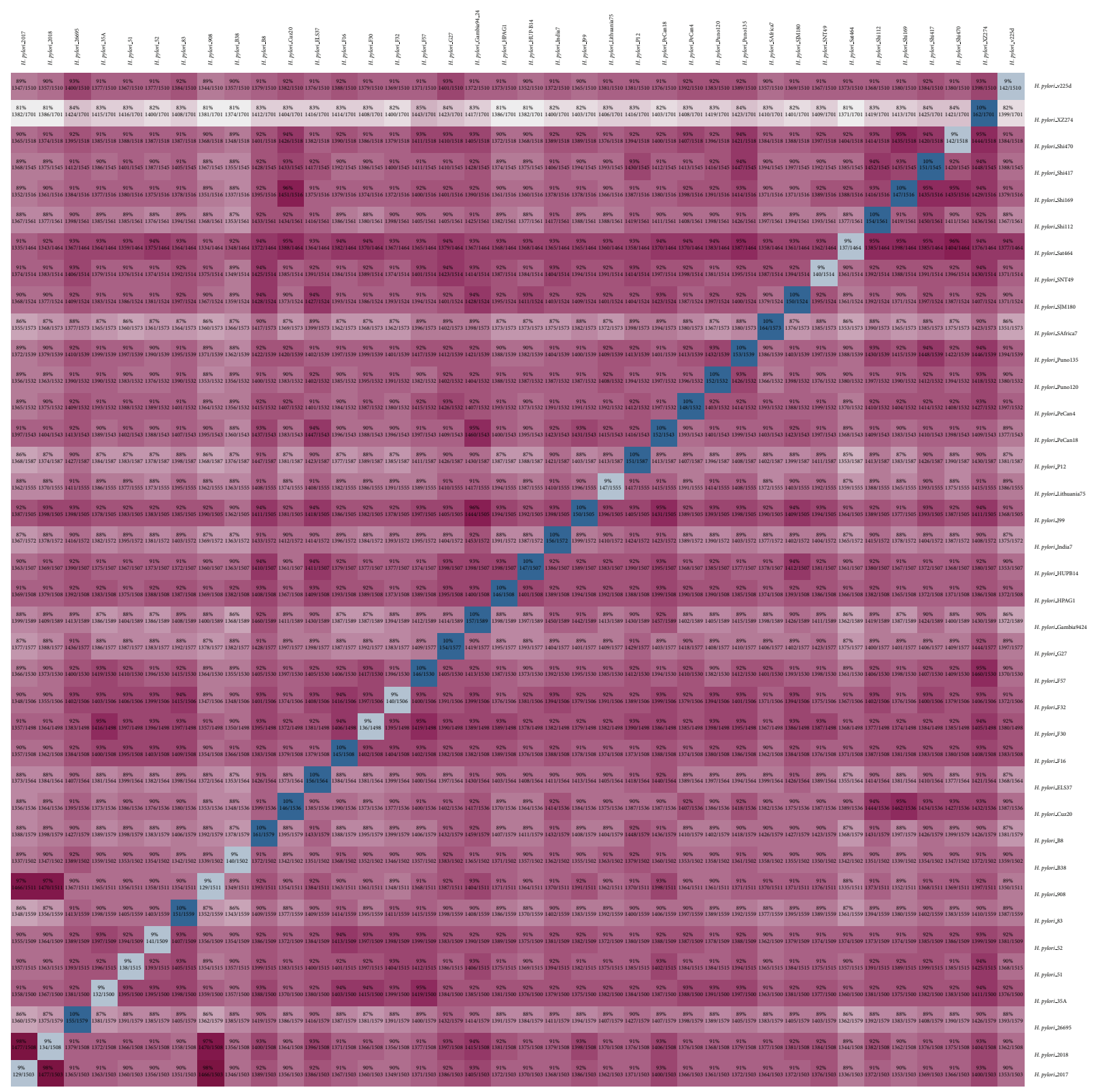

Homology between proteomes

80

99

(\%)

Homology within proteomes

8

(\%)

Figure 2: Whole genome/proteome pairwise alignment and comparative analysis. The translated genomes of all $H$. pylori available strains are analyzed by BLASTp analysis. Pairwise comparisons across $H$. pylori proteomes are plotted in blast matrix. The shared proteome between any two H. pylori genomes and the percentage of similarities is calculated and shown in corresponding boxes, where the color intensity indicated the similarity. The diagonal row of rectangular boxes in the matrix illustrates the internal homology against its own proteome. 


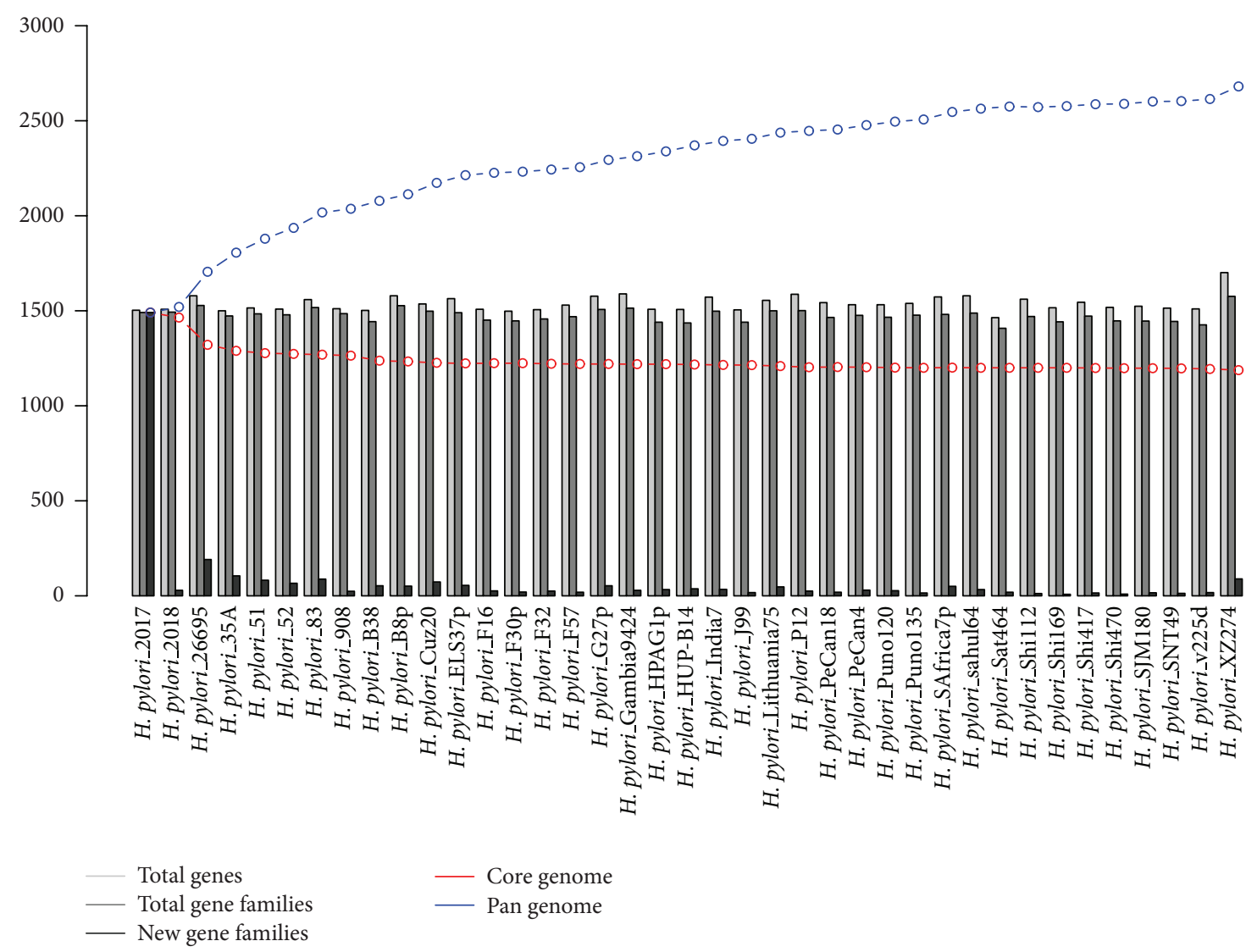

FIgure 3: Core and pan-genome estimation for the genus, $H$. pylori and non-pylori species. The figure demonstrates the distribution of core $(1,193)$ and pan-genome $(2,614)$ of $H$. pylori species (Table 1). The pan-genome plot represents total number of genes, gene clusters (families) for each genome (light grey), new gene families (dark gray) pan-genome (blue), and core genome (red). Name of genome is provided on $x$-axis and number of genes can be observed on $y$-axis.

interstrain relationships. For example, H. pylori 2017 and H. pylori 2018 are located quite close in phylogenetic tree; this also reflects the similar geographic relationship (West Africa). Similarly, they are closely related to $H$. pylori 908 , isolated from an African patient living in France having history of recrudescent duodenal ulcer disease, which in turn was found phylogenetically related to $H$. pylori J99, isolated from gastric carcinoma and gastritis patients in Costa Rica. These findings helped in understanding the intraspecies (strains) diversity and/or similarity and its correlation with that of phylogenomic variations based on genomic contents.

3.4. Pan-Genome Analysis of $H$. pylori. The global gene repertoire of $39 \mathrm{H}$. pylori genomes comprises 59,958 genes. Among this set of genes, core or central genome was estimated to contain 1,193 CGFs and the pan-genome was found to contain 2,614 PGF. An average genome of $H$. pylori contains $\sim 1537$ genes (the lowest 1,464 in H. pylori Sat 464 and the highest 1,701 in $H$. pylori XZ274). The calculated core genome represents $\sim 77 \%$ of the average genome size $(\sim 1,537$ genes/proteins). As expected, the $H$. pylori genomes (isolates) share a large part of their genomic content and a decrease in the number of new GFs/genes was observed as compared to what was accumulated upon subsequent genome inclusion to the study; this can be seen in the pan-core genome plot in Figure 3. We also observed accumulation of an average of $\sim 39$ new gene GFs (40 genes) with subsequent addition of genome; however, the highest numbers of species specific (unique) GFs observed were 189, 103, and 89 in H. pylori 26695, H. pylori 35A, and $H$. pylori XZ274, respectively (Table 1). These findings demonstrated that these particular strains are more diverse among those analyzed strains; the phylogenic tree also reflects similar image of evolutions or genome distributions. The pan-genome, on the other side, rose at a lower rate and roughly twice the size $(\sim 57 \%)$ of the core genome while the core genome is about $45 \%$ of the global gene repertoire, that is, the pan-genome of $H$. pylori. In a previous study by Salama et al., in 2000, they determined the core set of genes in $15 \mathrm{H}$. pylori strains with a microarray method, and a total of 1,281 genes were found to constitute the core genome [11]. As the strains were mainly isolated from only the western part of the world, it was difficult to estimate that those core genes would also represent the larger number of species, once they became available. However, it is not much surprising to estimate high number of CGFs for a few intraspecies genomes due to higher genomic similarities. On a random comparison, the first 15 genomes in our dataset represent $1,223 \mathrm{CGFs}$, which is close to the core genome $(1,281)$ previously observed. Furthermore, 25 genomes were found to share 1,208 CGFs and the total set of 
39 genomes constitutes a core genome of 1,193 CGFs (Additional File 1, in Supplementary material available online on http://dx.doi.org/10.1155/2014/139580). Our findings are quite closed to previous data, indicating the consistent behavior, evolution, and gene distribution $[11,40]$. Nevertheless, we are sure that even with the addition of more genome sequences, the pattern would remain the same or the conserved regions would be limited, but not necessary. Also, an average of $\sim 40$ new genes added to the study on addition of each new strain. The overall picture of $H$. pylori genomes statistics (genes, gene clusters (GFs), and unique genes associated with), core, and pan-genome pattern is shown in Figure 3, and the individual genomic data can be found in Table 1 .

\subsection{H. pylori Core EGFs and Nonhost Homologs Predictions.} Essential genes in a genome are the genes which are crucial for growth, cellular activities, and foundation of life. These genes represent a minimal gene set which is essential for a living cell. The identification of essential genes is of practical importance in drug designing against bacterial infections, and due to the fact that most of the antibiotics target essential cellular processes and essential gene products of microbial cells, they are suitable candidate targets for such drugs [43]. For this propose, we subjected our predicted CGFs $(1,193)$ against the database of essential genes, DEG and classified CGFs as EGFs if they had significant similarity against experimentally validated essential genes in other bacteria, bacterial group, or database. We predicted a total of $779(\sim 65 \%)$ EGFs in our core data set with the following selection parameters: $e$-value cutoff $10^{-10}$, percentage of identity $\geq 35 \%$ between query and hits, and a minimum bit score of 100 [27]. The list of those 779 EGFs along with their matching DEG ID is provided in Additional File 2. In principle, ideal targets for bacterial therapeutics are pathogen's genes/protein whose homologs are absent in the corresponding host. In the case of $H$. pylori, colonizing humans, we downloaded the human proteome (taxid: 9606) available on GenBank, which has 34,521 proteins. BLASTp analyses were performed and the predicted core EGFs in previous step (779) was aligned against human proteome. This time, the $e$-value cutoff was set to 0.005 and the percent of identity to $35 \%$. Proteins without hits below the set parameters were chosen as nonhost bacterial proteins. Two datasets were then generated and the so-called pathogen essential gene families having human homologs (497) were kept aside. The remaining 283 core essential proteins were found specific to pathogen (H. pylori) and do not have corresponding human homologs with same threshold (Additional File 3). The later set (core nHEGFs = 283) were selected as potential candidates and subjected to further validations (sequence and structural).

3.6. Vaccine Candidates Identification. For multiple related pathogenic species (intraspecies), once the complete genome sequences are available, it is a desirable task to predict global vaccine targets that are conserved among all genomes. Generally, surface and secreted proteins are involved in bacterial pathogenesis and are recognized as good candidates for vaccine development $[19,44]$. On the other hand, cytoplasmic and inner membrane proteins may not be good candidates for vaccine development; however, they can be targets for drug designing against bacteria. The following steps have been followed for the prediction of potential candidates.

3.7. Core Exoproteome Prediction. For H. pylori global vaccine identification, we analyzed our predicted nHEGFs set of EGFs (283) by an online protein localization tool CELLO [45]. Among the 283 (nHEGFs), 29 proteins predicted to be associated with bacterial cell surface ( 7 outer membrane, 12 inner membrane 8 periplasmic, and 5 extracellular) and some of the proteins were found to have multiple locations. Multifasta file containing predicted surface proteins (sequences) is created and is provided as Additional File 4.

3.8. Prioritization of Vaccine Targets. The predicted $29 \mathrm{H}$. pylori (CGFs/EGFs) surface proteins predicted by using CELLO [45] and PSORTb [46] were then analyzed for their molecular weight. Proteins having molecular weight $<110 \mathrm{KDa}$ are thought to be good targets for vaccine. nHEGFs generated in our analysis showed that 28 out of 29 proteins have molecular weight $<110 \mathrm{KDa}$ which are then supposed to be good targets for vaccines (Table 2 ).

3.9. Functional Annotation of Targets. Blas2go being a reliable workstation was used to generate functional annotation of targeted proteins [29]. Proteins were analyzed for their functions, characterization as enzymes, and InterPro scan. 28 out of 29 proteins were predicted to be potential therapeutic targets and are found to be involved in many essential biological functions such as abc transporter, membrane protein, intertase, and potassium transporter. Eleven core proteins are enzyme in nature and thus can be the best for vaccine or therapeutic targets. Complete functional annotation of proteins is given in Table 2. Blast2go was used to analyze molecular and biological function distribution among 28 proteins and a graph was obtained showing most of the proteins involved in hydrolase activity, organic cyclic compound binding, heterocyclic compound binding, substratespecific transporter activity, ion binding, and transmembrane transporter activity (Figure 4).

3.10. Protein-Protein Interaction Network. The 28 predicted potential surface core exoproteins were analyzed to generate a PPI network of $H$. pylori. The interaction map generated by STRING revealed many new insights of protein interactions among target proteins. In principle, the interaction network is constructed by integrating many types of experimentally proved statistics like cooccurrence, neighborhood, gene fusion, homology, text mining, and coexpression [30]. PPI map shown in Figure 5 exhibits direct or indirect relation between targeted proteins; 11 proteins appear to have direct relations/interactions among each other. Further enrichment analyses of proteins appearing in interactome have been carried out to explore potential role of these interactions; KEGG pathway analysis showed involvement of interacting proteins in citrate cycle, metabolic pathways, microbial metabolism in diverse environment, $\mathrm{ABC}$ transporters, and 
TABLE 2: Biological categorization of candidate essential gene families. The H. pylori EGFs analyzed by CELLO and PSORTb for prediction of surface proteins. This is followed by calculation of molecular weight of proteins by Expasy tool and Blast2go is used for functional annotation.

\begin{tabular}{|c|c|c|c|c|}
\hline Protein & Localization & Mol. weight (Da) & EC number & $\begin{array}{c}\text { Sequence } \\
\text { description }\end{array}$ \\
\hline SeqID: /gene_family="1495" & $\begin{array}{c}\text { Cytoplasmic } \\
\text { Outer membrane }\end{array}$ & 76937.16 & & \\
\hline SeqID: /gene_family="1623" & $\begin{array}{l}\text { Outer membrane } \\
\text { Inner membrane }\end{array}$ & 81336.76 & $\begin{array}{l}\text { EC 3.6.3.3, } \\
\text { EC 3.6.3.4, } \\
\text { EC 3.6.3.5 }\end{array}$ & Copper-exporting ATPase \\
\hline SeqID: /gene_family=“1487” & $\begin{array}{c}\text { Extracellular } \\
\text { Outer membrane }\end{array}$ & 51589.98 & EC 3.4.21 & Serine protease \\
\hline SeqID: /gene_family="1447" & $\begin{array}{c}\text { Outer membrane } \\
\text { Cytoplasmic }\end{array}$ & 24108.16 & & \\
\hline SeqID: /gene_family=" 821 " & $\begin{array}{l}\text { Inner membrane } \\
\text { Outer membrane }\end{array}$ & 26170.79 & & \\
\hline SeqID: /gene_family=“ $222 "$ & $\begin{array}{l}\text { Inner membrane } \\
\text { Cytoplasmic }\end{array}$ & 61901.64 & & Abc transporter permease \\
\hline SeqID: /gene_family=“197" & Inner membrane & 32817.21 & EC 2.5.1 & Prenyltransferase \\
\hline SeqID: /gene_family=“577” & Inner membrane & 25149.00 & & Membrane protein \\
\hline SeqID: /gene_family="1486" & Inner membrane & 62563.95 & & Insertase \\
\hline SeqID: /gene_family=“948” & Inner membrane & 52460.09 & & Lysine-specific permease \\
\hline SeqID: /gene_family="1028" & $\begin{array}{c}\text { Cytoplasmic } \\
\text { Inner membrane }\end{array}$ & 33346.74 & EC 1.1.1.8 & $\begin{array}{c}\text { Glycerol-3-phosphate } \\
\text { dehydrogenase }\end{array}$ \\
\hline SeqID: /gene_family="1439" & Inner membrane & 46137.77 & & Potassium transporter \\
\hline SeqID: /gene_family="1480" & Inner membrane & 53997.01 & & Sodium proline symporter \\
\hline SeqID: /gene_family=" $714 "$ & Inner membrane & 49579.70 & & $\begin{array}{l}\text { Sodium: neurotransmitter } \\
\text { symporter family protein }\end{array}$ \\
\hline SeqID: /gene_family="1238” & Inner membrane & 115481.83 & & Cation efflux system protein \\
\hline SeqID: /gene_family="230" & $\begin{array}{c}\text { Outer membrane } \\
\text { Extracellular }\end{array}$ & 30259.72 & EC 1.3.1.9 & Enoyl-acp reductase \\
\hline SeqID: /gene_family=“940" & Extracellular & 33739.71 & & \\
\hline SeqID: /gene_family=“792" & Extracellular & 39410.96 & & \\
\hline SeqID: /gene_family=“ $747 ”$ & $\begin{array}{c}\text { Cytoplasmic } \\
\text { Outer membrane } \\
\text { Extracellular }\end{array}$ & 32881.19 & & Domain protein \\
\hline SeqID: /gene_family=“30" & Periplasmic & 30271.1 & & 50s ribosomal protein 12 \\
\hline SeqID: /gene_family="1043" & $\begin{array}{l}\text { Periplasmic } \\
\text { Cytoplasmic }\end{array}$ & 15669.30 & & \\
\hline SeqID: /gene_family="128” & $\begin{array}{l}\text { Periplasmic } \\
\text { Cytoplasmic }\end{array}$ & 37289.58 & EC 3.5.1.49 & Formamidases \\
\hline SeqID: /gene_family=“ $428 ”$ & Periplasmic & 40467.09 & EC 1.1.1.1 & $\begin{array}{c}\text { NADP-dependent alcohol } \\
\text { dehydrogenase }\end{array}$ \\
\hline SeqID: /gene_family=“664" & $\begin{array}{l}\text { Cytoplasmic } \\
\text { Periplasmic }\end{array}$ & 26515.58 & EC 3.6.1.3 & $\begin{array}{l}\text { ABC transporter ATP-binding } \\
\text { protein }\end{array}$ \\
\hline SeqID: /gene_family=“928” & Periplasmic & 61103.25 & EC 2.3.2.2 & Gamma-glutamyltranspeptidase \\
\hline SeqID: /gene_family="1097" & Periplasmic & 65613.27 & EC 3.1.4.16 & 5-nucleotidase protein \\
\hline SeqID: /gene_family=“88" & Cytoplasmic membrane & 21533.23 & & Membrane protein \\
\hline SeqID: /gene_family=“1595" & Cytoplasmic membrane & 74214.44 & EC 2.41.129 & Penicillin-binding protein 1a \\
\hline SeqID: /gene_family=“387” & Periplasmic & 62296.56 & & $\begin{array}{l}\text { Peptide } \mathrm{ABC} \text { transporter } \\
\text { substrate-binding protein }\end{array}$ \\
\hline
\end{tabular}


Molecular function (level 3)

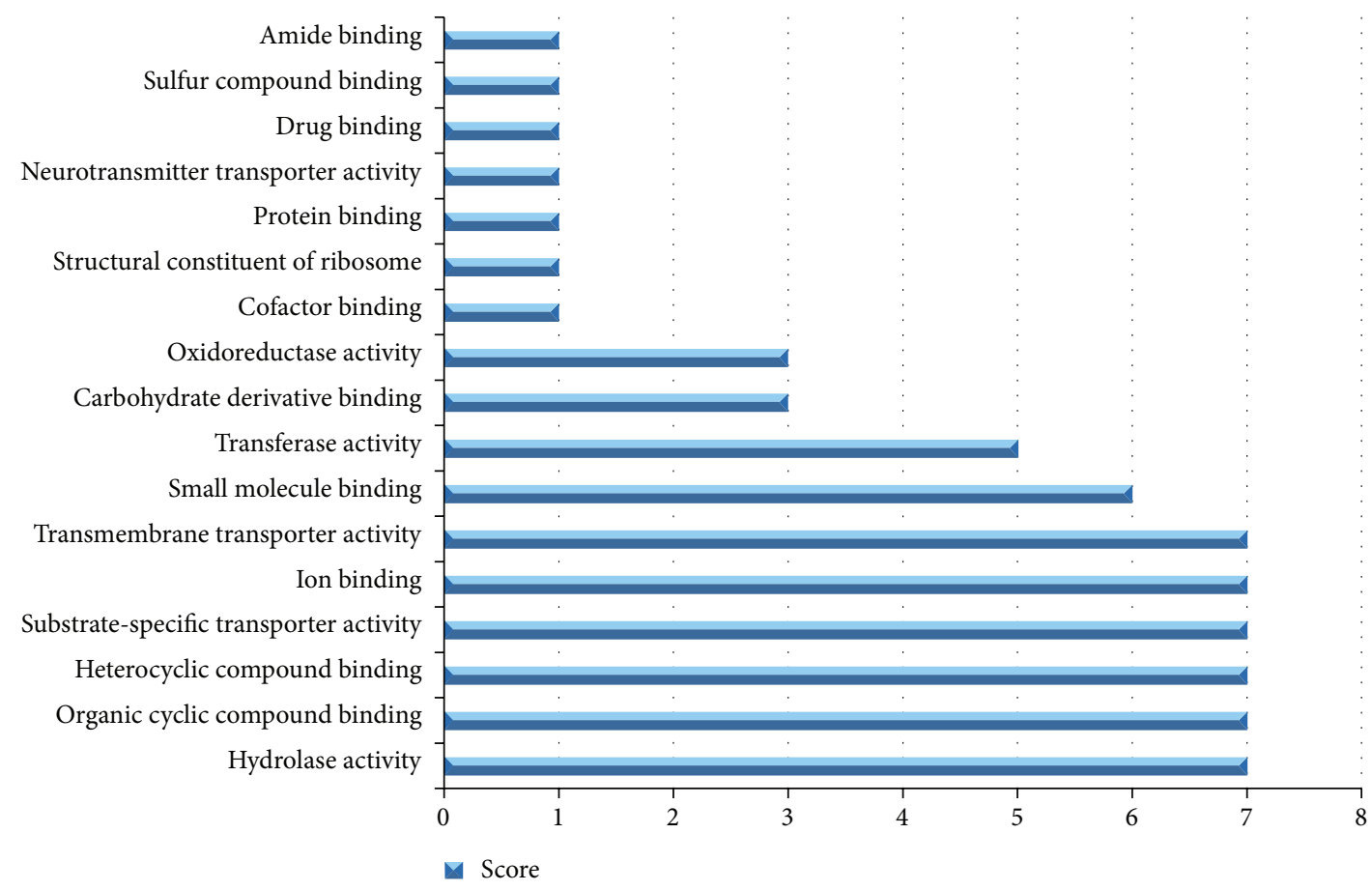

FIGURE 4: Functional characterization of proteins selected as potential vaccine targets. Total of 29 nonhuman homolog proteins were functionally annotated using Blast2go and distribution of their molecular functions in analyzed in the form of graph.

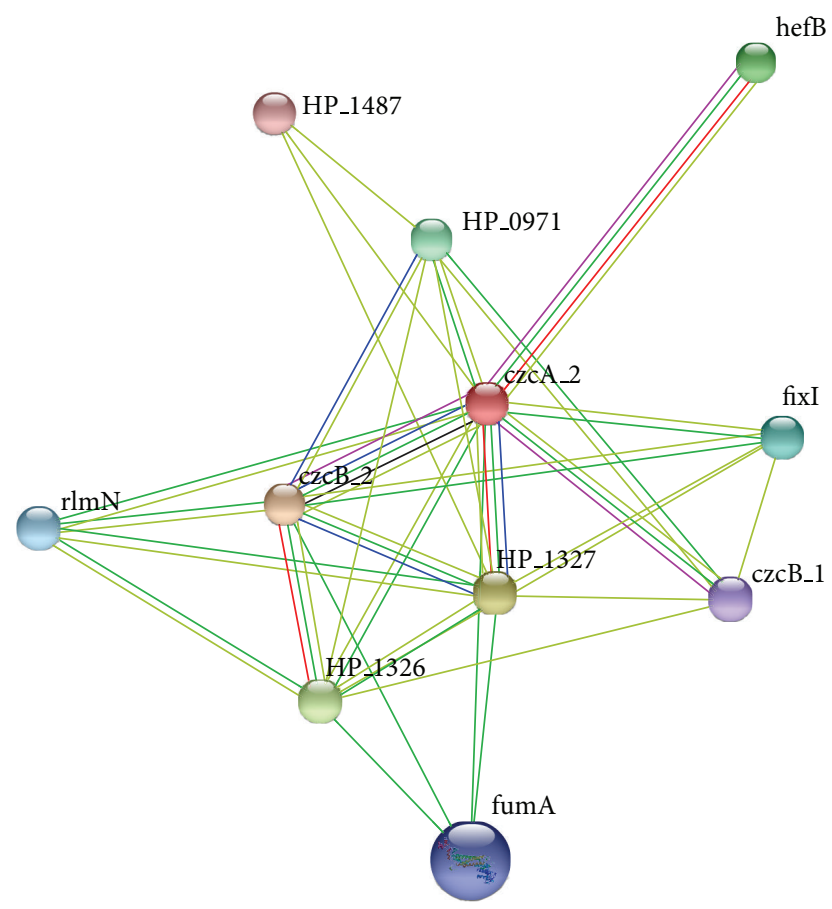

FIGURE 5: Protein-protein interaction (PPI) map of targeted core proteins. An interactome established between prioritized core protein targets. Eleven proteins showed interactions among each other revealing their collaborations in different pathways. Number and color of edges between nodes represent the type of evidence for the associations. various other metabolic pathways. Domain enrichment analysis revealed only 2 proteins having outer membrane efflux protein domain.

3.11. Epitope Mapping. The 28 potential proteins were then analyzed for epitopes which can bind to B-cells and generate immune response efficiently. BCPred was used to predict $75 \%$ specific 20-mer epitopes of each protein. Each protein has 1 or more sites that have ability to bind B-cells epitopes except a core protein 1028, a glycerol 3-phosphate dehydrogenase. Though it is an enzyme, no epitope was predicted in its sequence [31]. Three core proteins have a single epitope binding site, BCPred suggested 3 proteins with 2 sites for protective antigens, and all other core proteins have $>2$ epitope binding sites. All predicted epitopes were then subjected to VaxiJen to check their antigenic property (threshold = 0.4, ACC output). Being based on alignment independent method VaxiJen follows autocross covariance (ACC) algorithm that mines protein sequences for antigenicity based on chemical properties [32]. We used mode for bacterial protein prediction for protective antigens. Some proteins' epitopes do not show antigenic behavior whereas as some proteins show highly antigenic epitopes. Complete list of predicted epitopes and their VaxiJen score to be specified as antigenic or not are given in Additional File 5. Epitopes with highest scores for antigenicity can be further checked for MHC class binding and then can be approved as the best targets for vaccine development against $H$. pylori. Some epitopes from core proteins like MEHQNTTQKQGELKRDMKMR residing at position 
1 of gene_family = "948" (Additional File 5) have a VaxiJen score of 1.5091, which is much higher than the cutoff value 0.4, whereas some epitopes, for example, MMQLYKKHGANPLGGCLPLI initiating from 407 bp of gene_family = "1486," has 041 score near to cutoff value. Epitopes with the best value can be subjected to vaccine development. Although there are many loop holes in the defined experimental process of developing therapeutic targets, these in silico predicted epitopes can provide a good platform for vaccinology to begin a new era.

3.12. Pathogenicity Islands Prediction in H. pylori. We have identified 22 nonredundant putative PAIs across the genomes of H. pylori strains: 26695, Cuz-20, J99, PeCan4, and SouthAfrica7, which are named as putative pathogenicity islands of $H$. pylori 1-22 (PiHp1-22). To create a heatmap with the percentage of similarity of each PAI in all genomes, the content of PAIs identified as PiHp1-18, PiHp19, PiHp20-21, and $\mathrm{PiHp} 22$ were acquired from the genome sequences of H. pylori strains 26695, Cuz-20, SouthAfrica7, and PeCan4, respectively. Although some of the reference PAIs may be partially or totally present in the other reference genomes, these reference PAIs were chosen due to the higher representability (according to their size and gene content); for example, PiHp19 in H. pylori Cuz20 is also present in H. pylori 26695; however, it has only $40 \%$ of the genes of $\mathrm{PiHp} 19$ of $H$. pylori Cuz20 and, thus, the latter one has been chosen for comparative analysis.

In the heatmap, we observed a high degree of variability in most of the PAIs across all 39 genomes, where only PiHps 2 (C694_01095-C694_01190), PiHps 4 (C694_01445C694_01490), PiHps 14 (C694_05735-C694_05795), and PiHps 15 (C694_06365-C694_06390) are totally present in at least $50 \%$ of the strains (Figure 6). On the other hand, PiHps 8 (C694_02175-C694_02345) and PiHps 13 (C694_05045-C694_05230) are the most variable regions, presenting percentages of similarity ranging from $0-57 \%$ to $10-71 \%$, respectively. Figure 7 demonstrates the conserved PiHps 2, 4, 14, and 15 (Figures 7(a), 7(b), 7(c), and 7(d)) and variable PAIs PiHps 8, 13, and 9 (Figures 7(e), 7(f), and $7(\mathrm{~g}))$.

The majority of the genes harbored by PiHps 2, 4, 8, 13, 14 , and 15 have been assigned the term "hypothetical protein" on their product tag, meaning that most of the gene products are not yet identified. However, some genes on these PAIs deserve attention; for example, $\mathrm{PiHp} 2$ harbors a gene coding for a DNA repair protein, $\operatorname{RadA}$ (C694_01125), which is part of a superfamily of recombinases or DNA strand-exchange proteins, composed of archaeal RadA, bacterial RecA, and eukaryal Rad51 and DMC1 proteins. RadA has a pivotal role in DNA strand-exchange process between single stranded DNA (ssDNA) and a homologous double-stranded DNA (ds-DNA) in homologous recombination [47]. Homologous recombination is one of several DNA repair pathways (direct reversal, base excision repair, nucleotide excision repair, mismatch repair, and recombination repair pathways) and functions in the repair of double-stranded DNA breaks and the restarting of stalled replication forks, therefore, guar- anteeing accurate functioning and propagation of genetic information $[47,48]$. However, as $\operatorname{RadA}$ is mainly found in archaea, in vitro experiments are required to elucidate the putative function of RadA in bacteria, specially $H$. pylori and to clarify its putative acquirement through horizontal gene transfer from archaea.

In $\mathrm{PiHp} 4$, there are genes coding for a cell division protein FtsH (C694_01445) and a toxin-like outer membrane protein (C694_01460), also termed putative vacuolating cytotoxin(VacA) like protein. The cell division protein $\mathrm{FtsH}$ is a member of the AAA+ super-family (ATPases associated with diverse cellular activities), which consists of highly conserved molecular machines responsible for a number of cellular processes like cell division, cell differentiation, signal transduction, stress response, and others [49]. FtsH is required for the proper functioning of sigma 54 under nitrogen limitation conditions and $\mathrm{FtsH}$ mediated degradation of misassembled membrane and cytoplasmic proteins is thought to be responsible for its role in the heat shock response, therefore explaining its upregulation in response to heat shock in several bacteria, including $H$. pylori $[50,51]$. VacA, or vacuolating cytotoxin A, is one of the most studied toxins produced by H. pylori, whereas the CagA (cytotoxinassociated gene A) occupies the first place. VacA has the ability to cause vacuole-like membrane vesicles in the cytoplasm of gastric cells and is also associated with disruption of mitochondrial functions, stimulation of apoptosis, and blockade of T-cell proliferation. The presence of the toxigenic allelic s1 form of VacA in strains of $H$. pylori is commonly associated with an increased risk of peptic ulceration and gastric cancer [7, 52-54]. CagA, on the other hand, after injected in host cells, can influence cellular tight junction, cellular polarity, cell proliferation and differentiation, cell scattering, and induction of the inflammatory response. CagA was already identified to be located in an extensively studied pathogenicity island (cag-PAI), which is $\sim 40 \mathrm{~kb}$ in size, encodes for a type IV secretion system (T4SS), and was identified by PIPS, in this work, as PiHp9 (C694_02670C694_02870) (for detailed reviews of VacA and CagA, please refer to $[52,54])$.

Inside the most variable PAIs, PiHps 8 and 13, IS605 transposases tnpA (C694_02220, C694_05100 and C694_05150) and tnpB (C694_02225, C694_05105 and C694_05145) can be observed, which are probably responsible for the incorporation of the PAIs and could also account for their high instability. Additionally, $\mathrm{PiHp} 8$ also harbors genes coding for 2 VirB4-like proteins (C694_02240 and C694_02345). VirB4 is part of T4SS in bacteria, which is necessary for pilus biogenesis, substrate transfer, and virulence as it is responsible for horizontal transfer of plasmid DNA between bacteria during conjugation and for the delivering of macromolecules to prokaryotes and eukaryotes $[55,56]$. In $H$. pylori, the T4SS formed by the VirD4/VirB genes are known to have a pivotal role in the delivery of CagA protein into the cytosol of gastric epithelial cells and the entire T4SS is coded by VirD4/VirB genes harbored by the cag-PAI (PiHp9). The putative backup function of the 2 copies of VirB4-like proteins in PiHp8 for the VirB4 harbored by cag-PAI is yet to be elucidated. 


\begin{tabular}{|c|c|c|c|c|c|c|c|c|c|c|c|c|c|c|c|c|c|c|c|c|c|c|}
\hline & 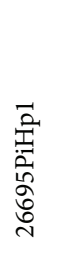 & 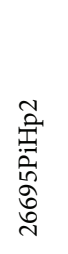 & 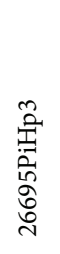 & 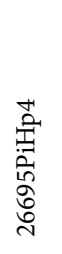 & 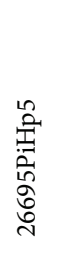 & 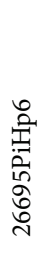 & 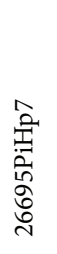 & 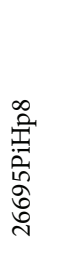 & 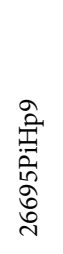 & 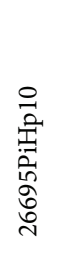 & 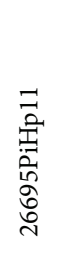 & 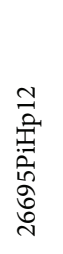 & 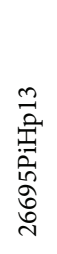 & 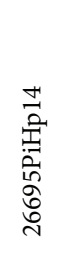 & 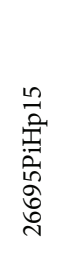 & 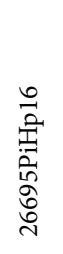 & 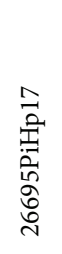 & 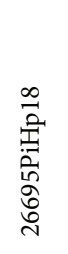 & 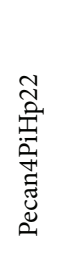 & 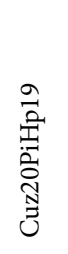 & 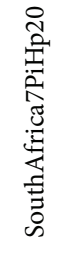 & $\overrightarrow{\widetilde{N}}$ \\
\hline rica7 & 92 & 0 & 95 & 88 & 100 & 68 & 94 & 29 & 24 & 82 & 94 & 77 & 43 & 100 & 100 & 89 & 79 & 87 & 81 & 60 & 100 & 100 \\
\hline$n 4$ & 5 & 1 & 95 & 00 & 94 & - & 88 & 29 & 97 & 82 & 81 & 77 & 43 & 100 & 100 & 8 & 6 & 37 & 00 & 51 & 45 & \\
\hline Puno120 & 0 & & 90 & 88 & 94 & 7 & 76 & 25 & 91 & 91 & 94 & 77 & 33 & 100 & 100 & 57 & 9 & 37 & 76 & 33 & 15 & \\
\hline $\mathrm{P}$ & 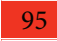 & 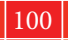 & 90 & 00 & 94 & 77 & 82 & 29 & 97 & 91 & 94 & 77 & 48 & 100 & 100 & 39 & 36 & 30 & 31 & 51 & 15 & \\
\hline $5 \mathrm{~d}$ & 2 & 14 & 86 & 0 & 88 & 1 & 82 & 32 & 97 & 91 & 88 & 85 & 14 & 91 & 100 & 39 & 9 & 87 & 31 & 31 & 5 & \\
\hline Cuz20 & 85 & 4 & 90 & 8 & 94 & 82 & 88 & 7 & 94 & 82 & 94 & 92 & 43 & 91 & 100 & 67 & 36 & 87 & 81 & 00 & 15 & \\
\hline Shi169 & 33 & 0 & 95 & 0 & 94 & 82 & 8 & & $\overline{94}$ & 91 & 94 & 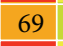 & 38 & - & 100 & 39 & 6 & 30 & & 60 & 45 & \\
\hline Shil12 & 30 & 0 & 95 & 0 & 94 & 64 & 82 & 11 & 91 & 91 & 81 & 92 & 48 & 100 & 100 & 6 & 79 & 87 & 1 & 2 & 6 & \\
\hline & 90 & 0 & 95 & & 94 & 8 & 88 & 36 & 94 & 82 & 88 & 85 & 52 & 91 & 100 & & 93 & 93 & 81 & 58 & 5 & \\
\hline Shi470 & 87 & $\underline{-1}$ & 95 & 0 & 88 & 82 & 82 & 7 & 97 & 73 & 88 & 8. & 43 & 100 & \begin{tabular}{|l|}
100 \\
\end{tabular} & 89 & 79 & 87 & 81 & 63 & 5 & \\
\hline Sat464 & 87 & $\overline{4}$ & 95 & 0 & 94 & 82 & 82 & 4 & 70 & 82 & 88 & 7 & 24 & 100 & 100 & 78 & 36 & 87 & 31 & 31 & 45 & \\
\hline 83 & 92 & 94 & 95 & 0 & 10 & 86 & 9. & 11 & 100 & 100 & 94 & 85 & 33 & 100 & 1 & & 93 & 93 & 00 & 8 & 15 & \\
\hline F16 & 92 & 100 & 95 & 88 & 94 & 7 & 88 & ${ }^{2}$ & 97 & 91 & 94 & 8 & 14 & 100 & 100 & 39 & 93 & 93 & 81 & 41 & 5 & \\
\hline F57 & 92 & 84 & 95 & $8 \varepsilon$ & 94 & 82 & $8 \varepsilon$ & 2 & 94 & 91 & 9. & 7 & 43 & 0 & & & & 93 & & & 55 & \\
\hline $35 \mathrm{~A}$ & 85 & 94 & 81 & 8 & 94 & 77 & 76 & 14 & 100 & 00 & 88 & 62 & 10 & 100 & 100 & 67 & 9 & 93 & 81 & 31 & 55 & \\
\hline F30 & 92 & 0 & 95 & 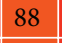 & 94 & 82 & $=$ & 14 & 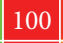 & 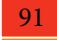 & 94 & 9 & & 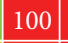 & & & & & & & 5 & \\
\hline 51 & 83 & 4 & 95 & 8 & 94 & 73 & 88 & 7 & 100 & 73 & 94 & 62 & 38 & 91 & 100 & 9 & 79 & 93 & 31 & 36 & 55 & 3 \\
\hline F32 & 90 & $=$ & 95 & & 94 & & $8 \varepsilon$ & 11 & & & 9 & & 38 & 100 & & & & & & & & \\
\hline 52 & 92 & 94 & 95 & 0 & 94 & 73 & 88 & 0 & 100 & 82 & 88 & 85 & 10 & 100 & 100 & 89 & 79 & 93 & 76 & 28 & 15 & 4 \\
\hline 274 & 83 & & 81 & & 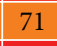 & & 00 & 32 & or & 73 & 81 & & 48 & 10 & 10 & & & & & & 55 & \\
\hline IT49 & 85 & 94 & 95 & 100 & 100 & 64 & 76 & 7 & 97 & 73 & 94 & 85 & 52 & 11 & 100 & 56 & 86 & 73 & 81 & 59 & 65 & \\
\hline India7 & 92 & 1 & \begin{tabular}{|l|}
95 \\
\end{tabular} & & 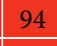 & - & $8<$ & 11 & 74 & 82 & 88 & 77 & 52 & 100 & 10 & & & | & 4 & 10 & 5 & \\
\hline ia94/24 & 92 & $\overline{94}$ & 90 & & 94 & 6 & 82 & 11 & 94 & 82 & 88 & 6 & 62 & 100 & 100 & 89 & 86 & 87 & 81 & 74 & 55 & 30 \\
\hline 7 & 90 & & 90 & & y. & 64 & 100 & 0 & 100 & 82 & 81 & 6. & 1 & 82 & 11 & 8 & 93 & 53 & 81 & 43 & 55 & \\
\hline 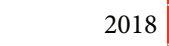 & 93 & & 95 & & 94 & & 94 & 0 & 100 & 82 & 8 & & 19 & 82 & 100 & & 93 & 53 & $\overline{81}$ & & 15 & 4 \\
\hline 908 & 93 & 94 & 95 & & 8 & 6 & 94 & 4 & 97 & 8 & 81 & 62 & 19 & 82 & & & 93 & 53 & 81 & 43 & 5 & \\
\hline J99 & 92 & & -1 & & 88 & & 04 & 0 & 100 & & 94 & & 33 & - & 100 & & & 93 & 81 & & 55 & 1 \\
\hline Pecan18 & 90 & & 95 & 15 & 94 & 64 & 82 & 11 & 97 & 82 & 88 & - & 43 & 100 & 100 & Ir & 71 & 73 & 81 & 34 & 55 & 2 \\
\hline LS37 & 92 & & 86 & & $\sqrt{21}$ & 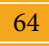 & 102 & 11 & 94 & 72 & 81 & - & 3 & 100 & 100 & & & 93 & 81 & 37 & 33 & 57 \\
\hline HUP-B14 & 92 & 89 & 95 & & 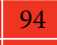 & 6 & 76 & c & 91 & 8 & 88 & 1 & 29 & 100 & 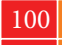 & 10 & 79 & 87 & 81 & 30 & 73 & 50 \\
\hline SJM180 & 92 & & 90 & & $\overline{94}$ & 6 & 82 & -1 & 97 & 82 & 94 & 54 & 43 & 100 & 100 & 78 & 86 & 100 & 81 & 38 & 64 & 59 \\
\hline B8 & 95 & 9. & 95 & & 9. & 59 & 88 & 32 & 100 & 91 & 94 & 92 & 62 & 100 & 100 & 100 & 93 & 87 & 81 & 47 & 91 & 72 \\
\hline PAG1 & 93 & 100 & 95 & 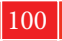 & $2=$ & 1 & 82 & 0 & 100 & 91 & 94 & 85 & 10 & 100 & 100 & 89 & 93 & 93 & 81 & 31 & 55 & 54 \\
\hline P12 & 98 & 94 & 9 & & 94 & 8 & 88 & 57 & 100 & 9 & 94 & 92 & 71 & 100 & 100 & 78 & 93 & 87 & 81 & 50 & 55 & 78 \\
\hline 26695 & 100 & 100 & 100 & 1 & 100 & 100 & 100 & 100 & \begin{tabular}{|l|}
100 \\
\end{tabular} & 100 & 100 & 100 & 100 & 100 & 100 & $100 \mid$ & 100 & \begin{tabular}{|l|}
$\mid 100$ \\
\end{tabular} & 81 & 40 & 55 & 61 \\
\hline B38 & 88 & 89 & 86 & 1 & 94 & 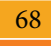 & 88 & 21 & 24 & 73 & 88 & 7 & 15 & 91 & 10 & 89 & 86 & o/ & 81 & 33 & 55 & 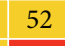 \\
\hline G27 & 90 & 94 & 95 & 100 & 74 & 11 & 88 & 25 & 94 & 82 & 94 & 85 & 57 & 91 & \begin{tabular}{|l|}
100 \\
\end{tabular} & 89 & 86 & 80 & 81 & 57 & 55 & 91 \\
\hline & & & & & 94 & & & & & & $y^{4}$ & & & & & 57 & 93 & U & & 3 & & \\
\hline
\end{tabular}

\begin{tabular}{|c|c|}
\hline 0 & $\geq 50$ \\
\hline$>0$ & $\geq 60$ \\
\hline$\geq 10$ & $\geq 70$ \\
\hline$\geq 20$ & $\geq 80$ \\
\hline$\geq 30$ & $\geq 90$ \\
\hline$\geq 40$ & 100 \\
\hline
\end{tabular}

FIgURE 6: Pathogenicity islands in H. pylori genomes (Pan-Heatmap). Heatmap analysis demonstrates high degree of variability on most of the PAIs across all genomes. Among the 22 predicted PAIs, only PiHps 2, 4, 14, and 15 are present in at least $50 \%$ of the strains. 


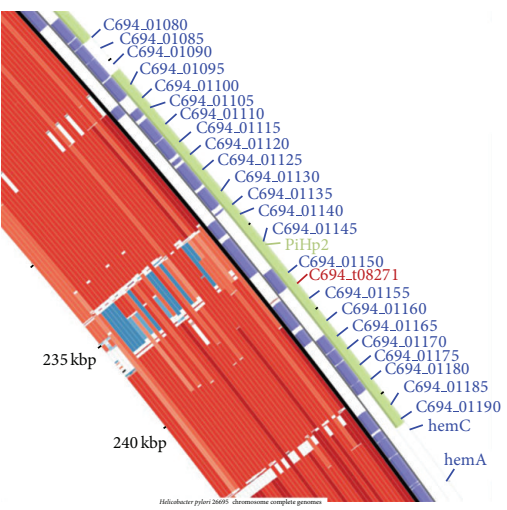

(a)

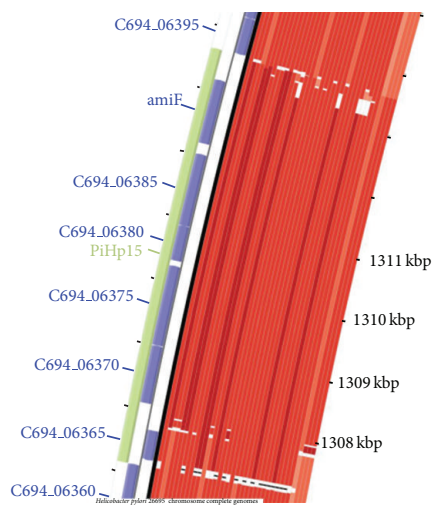

(d)

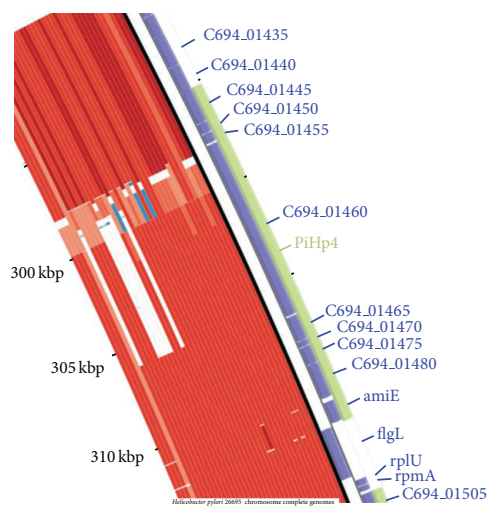

(b)

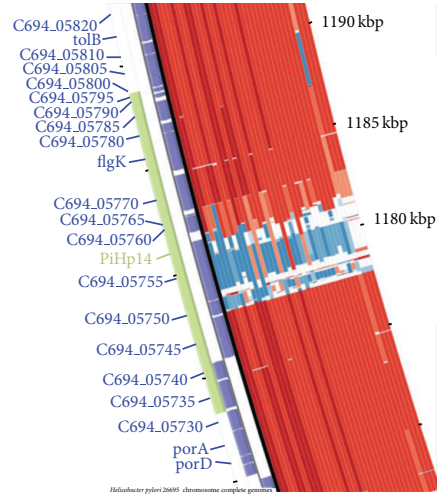

(c)

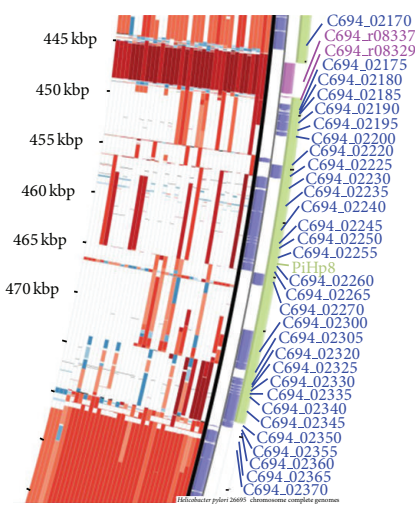

(e)



(f)

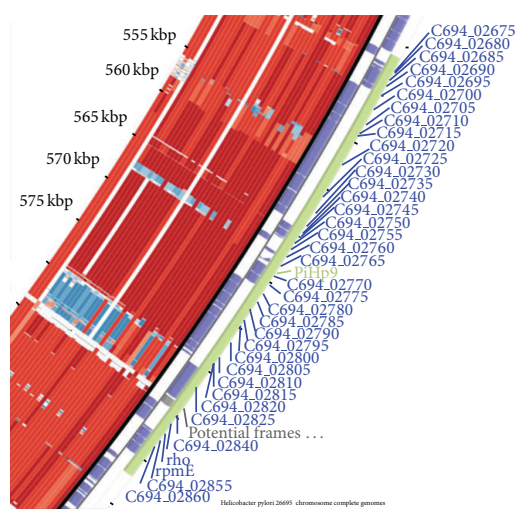

(g)

Figure 7: Conserved and variable pathogenicity islands in $H$. pylori. Putative pathogenicity islands predicted in H. pylori. The H. pylori 26695 a reference genome is selected for analysis (scaffold). All the genomes are aligned and a phylogenetically related nonpathogenic organism Wolinella succinogenes DSM 1740 is also included for comparison. PAIs 2, 4, 14, and 15 are found conserved ((a), (b), (c), and (d)). On the other side, PAIs PiHps 8, 13, and 9 ((e), (f), and (g)) were found variable among $H$. pylori genomes.

\section{Conclusion}

The study illustrates the comparative genomic and pangenomic analysis of important human pathogen $H$. pylori. Multiple genomes from the same species have been analyzed, showing genomic similarities to a large extent, and thus constitute relatively higher conserved core genome. The number of strain specific genes was observed to be significantly low, indicative of the close relationships among these strains. A number of potential novel pathogenicity islands were predicted and vaccine candidates and drug targets have been characterized providing detailed insights into the pathogenesis of $H$. pylori. Therapeutic targets' prioritization includes molecular weight analysis, nonhomology with host, and epitope mapping. Proteins with weight $<110 \mathrm{kDa}$ are supposed to be good targets for vaccines as they can easily be extracted and purified. Antigenic epitopes of selected core proteins can provide good insight into development of targeted peptide vaccines. The candidate genes and proteins identified could be further analyzed for the development of 
therapeutics against this neglected pathogen. Furthermore, conserved pathogenic regions in multiple genomes which have been identified will help in understanding the common pathogenic behavior of this pathogen. Finally, the comparative genomic tools and technique applied in the study can be extended to other pathogens even on a larger scale. We believe that the data generated during this study can assist further research in comparative genome analysis in order to expose the genetic markers of virulence, organism adaptability to host tissues, antibiotic resistance, and effective therapeutic strategies in the same or other similar species.

\section{Conflict of Interests}

The authors declared that there is no conflict of interests regarding the publication of this paper.

\section{Authors' Contribution}

Amjad Ali designed the study, collected data, and did the comparative analyses. Siomar C. Soares provided input in pathogenomics. Anam Naz, Marriam Bakhtiar, Sandeep Tiwari, Shah Hassan, Fazal Hanan, and Anderson Miyoshi assisted in therapeutic target analyses. Amjad Ali, Anam Naz, Marriam Bakhtiar, Fazal Hanan, David W. Ussery, Debmalya Barh, Anderson Miyoshi, Artur Silva, and Vasco Azevedo were involved in writing the paper. All authors read and approved the final paper.

\section{Acknowledgment}

The authors thank The World Academy of Sciences for the advancement of science in developing countries (TWAS), National Council for Scientific and Technological Development $(\mathrm{CNPq})$ for the award of the Ph.D. fellowship.

\section{References}

[1] P. Lehours, F. F. Vale, M. K. Bjursell et al., "Genome sequencing reveals a phage in Helicobacter pylori," mBio, vol. 2, no. 6, 2011.

[2] Y. You, L. He, M. Zhang et al., "Comparative genomics of helicobacter pylori strains of china associated with different clinical outcome," PLoS ONE, vol. 7, no. 6, Article ID e38528, 2012.

[3] Q.-J. Dong, Q. Wang, Y. Xin, N. Li, and S.-Y. Xuan, "Comparative genomics of Helicobacter pylori," World Journal of Gastroenterology, vol. 15, no. 32, pp. 3984-3991, 2009.

[4] S. Zhang, L. Moise, and S. F. Moss, "H. pylori vaccines: why we still don't have any," Human Vaccines, vol. 7, no. 11, pp. 1153-1157, 2011.

[5] T. P. Mikkonen, R. I. Kärenlampi, and M. Hänninen, "Phylogenetic analysis of gastric and enterohepatic Helicobacter species based on partial HSP60 gene sequences," International Journal of Systematic and Evolutionary Microbiology, vol. 54, no. 3, pp. 753-758, 2004.

[6] H. Gressmann, B. Linz, R. Ghai et al., "Gain and loss of multiple genes during the evolution of Helicobacter pylori," PLoS Genetics, vol. 1, no. 4, article e43, 2005.
[7] K. R. Jones, J. M. Whitmire, and D. S. Merrell, "A tale of two toxins: Helicobacter pylori CagA and VacA modulate host pathways that impact disease," Frontiers in Microbiology, vol. 1, Article ID Article 115, p. 115, 2010.

[8] M. R. Amieva, R. Vogetmann, A. Covacci, L. S. Tompkins, W. J. Nelson, and S. Falkow, "Disruption of the epithelial apicaljunctional complex by Helicobacter pylori CagA," Science, vol. 300, no. 5624, pp. 1430-1434, 2003.

[9] T. G. Blanchard, S. J. Czinn, P. Correa et al., "Genome sequences of 65 Helicobacter pylori strains isolated from asymptomatic individuals and patients with gastric cancer, peptic ulcer disease, or gastritis," Pathogens and Disease, vol. 68, pp. 39-43, 2013.

[10] N. R. Salama, G. Gonzalez-Valencia, B. Deatherage et al., "Genetic analysis of Helicobacter pylori strain populations colonizing the stomach at different times postinfection," Journal of Bacteriology, vol. 189, no. 10, pp. 3834-3845, 2007.

[11] N. Salama, K. Guillemin, T. K. McDaniel, G. Sherlock, L. Tompkins, and S. Falkow, "A whole-genome microarray reveals genetic diversity among Helicobacter pylori strains," Proceedings of the National Academy of Sciences of the United States of America, vol. 97, no. 26, pp. 14668-14673, 2000.

[12] R. Zhang and Y. Lin, "DEG 5.0, a database of essential genes in both prokaryotes and eukaryotes," Nucleic Acids Research, vol. 37, no. 1, pp. D455-D458, 2009.

[13] M. L. Acencio and N. Lemke, "Towards the prediction of essential genes by integration of network topology, cellular localization and biological process information," BMC Bioinformatics, vol. 10, article 290, 2009.

[14] C. Friis, L. J. Jensen, and D. W. Ussery, "Visualization of pathogenicity regions in bacteria," Genetica, vol. 108, no. 1, pp. 47-51, 2000.

[15] G. Zanotti, "Molecular aspects of Helicobacter pylori cagpathogenicity island," The FEBS Journal, vol. 278, no. 8, p. 1189, 2011.

[16] K. Lagesen, P. Hallin, E. A. Rødland, H. Stærfeldt, T. Rognes, and D. W. Ussery, "RNAmmer: consistent and rapid annotation of ribosomal RNA genes," Nucleic Acids Research, vol. 35, no. 9, pp. 3100-3108, 2007.

[17] D. Hyatt, P. F. Locascio, L. J. Hauser, and E. C. Uberbacher, "Gene and translation initiation site prediction in metagenomic sequences," Bioinformatics, vol. 28, no. 17, Article ID bts429, pp. 2223-2230, 2012.

[18] J. D. Thompson, D. G. Higgins, and T. J. Gibson, "CLUSTAL $\mathrm{W}$ : improving the sensitivity of progressive multiple sequence alignment through sequence weighting, position-specific gap penalties and weight matrix choice," Nucleic Acids Research, vol. 22, no. 22, pp. 4673-4680, 1994.

[19] A. Ali, S. C. Soares, A. R. Santos et al., "Campylobacter fetus subspecies: Comparative genomics and prediction of potential virulence targets," Gene, vol. 508, no. 2, pp. 145-156, 2012.

[20] N. Saitou and M. Nei, "The neighbor-joining method: a new method for reconstructing phylogenetic trees," Molecular Biology and Evolution, vol. 4, no. 4, pp. 406-425, 1987.

[21] K. Tamura, G. Stecher, D. Peterson, A. Filipski, and S. Kumar, "MEGA6: molecular evolutionary genetics analysis version 6.0," Molecular Biology and Evolution, vol. 30, pp. 2725-2729, 2013.

[22] O. Lukjancenko, D. W. Ussery, and T. M. Wassenaar, "Comparative genomics of Bifidobacterium, Lactobacillus and related 
probiotic genera," Microbial Ecology, vol. 63, no. 3, pp. 651-673, 2012.

[23] P. Leekitcharoenphon, O. Lukjancenko, C. Friis, F. M. Aarestrup, and D. W. Ussery, "Genomic variation in Salmonella enterica core genes for epidemiological typing," BMC Genomics, vol. 13, no. 1, article 88, 2012.

[24] E. Trost, J. Blom, S. C. de Soares et al., "Pangenomic study of Corynebacterium diphtheriae that provides insights into the genomic diversity of pathogenic isolates from cases of classical diphtheria, endocarditis, and pneumonia," Journal of Bacteriology, vol. 194, no. 12, pp. 3199-3215, 2012.

[25] A. Ali, S. C. Soares, E. Barbosa, A. R. Santos, D. Barh, and S. M. Bakhtiar, "Microbial comparative genomics: an overview of tools and insights into the genus corynebacterium," Journal of Bacteriology \& Parasitology, vol. 4, pp. 1-16, 2013.

[26] L. Snipen, T. Almøy, and D. W. Ussery, "Microbial comparative pan-genomics using binomial mixture models," BMC Genomics, vol. 10, article 385, 2009.

[27] A. M. Butt, I. Nasrullah, S. Tahir, and Y. Tong, "Comparative genomics analysis of mycobacterium ulcerans for the identification of putative essential genes and therapeutic candidates," PLoS ONE, vol. 7, no. 8, Article ID e43080, 2012.

[28] M. R. Wilkins, E. Gasteiger, A. Bairoch et al., "Protein identification and analysis tools in the ExPASy server," Methods in Molecular Biology, vol. 112, pp. 531-552, 1999.

[29] A. Conesa and S. Götz, "Blast2GO: a comprehensive suite for functional analysis in plant genomics," International Journal of Plant Genomics, vol. 2008, Article ID 619832, 12 pages, 2008.

[30] D. Szklarczyk, A. Franceschini, M. Kuhn et al., "The STRING database in 2011: functional interaction networks of proteins, globally integrated and scored," Nucleic Acids Research, vol. 39, no. 1, pp. D561-D568, 2011.

[31] Y. El-Manzalawy, D. Dobbs, and V. Honavar, "Predicting flexible length linear B-cell epitopes.," Computational systems bioinformatics / Life Sciences Society. Computational Systems Bioinformatics Conference, vol. 7, pp. 121-132, 2008.

[32] I. A. Doytchinova and D. R. Flower, "VaxiJen: a server for prediction of protective antigens, tumour antigens and subunit vaccines," BMC Bioinformatics, vol. 8, article 4, 2007.

[33] S. C. Soares, V. A. C. AbreuViní, R. T. J. Ramos et al., "PIPS: pathogenicity island prediction software," PLoS ONE, vol. 7, no. 2, Article ID e30848, 2012.

[34] C. Baar, M. Eppinger, G. Raddatz et al., "Complete genome sequence and analysis of Wolinella succinogenes," Proceedings of the National Academy of Sciences of the United States of America, vol. 100, no. 20, pp. 11690-11695, 2003.

[35] T. J. Carver, K. M. Rutherford, M. Berriman, M. Rajandream, B. G. Barrell, and J. Parkhill, "ACT: the Artemis comparison tool," Bioinformatics, vol. 21, no. 16, pp. 3422-3423, 2005.

[36] J. F. Tomb, O. White, A. R. Kerlavage et al., "The complete genome sequence of the gastric pathogen Helicobacter pylori," Nature, vol. 388, pp. 539-547, 1997.

[37] M. Eppinger, C. Baar, B. Linz et al., "Who ate whom? Adaptive Helicobacter genomic changes that accompanied a host jump from early humans to large felines," PLoS Genetics, vol. 2, no. 7, p. e120, 2006.

[38] S. Kutter, R. Buhrdorf, J. Haas, W. Schneider-Brachert, R. Haas, and W. Fischer, "Protein subassemblies of the Helicobacter pylori cag type IV secretion system revealed by localization and interaction studies," Journal of Bacteriology, vol. 190, no. 6, pp. 2161-2171, 2008.

[39] J. D. Oh, H. Kling-Bäckhed, M. Giannakis et al., "The complete genome sequence of a chronic atrophic gastritis Helicobacter pylori strain: evolution during disease progression," Proceedings of the National Academy of Sciences of the United States of America, vol. 103, no. 26, pp. 9999-10004, 2006.

[40] W. Lu, M. J. Wise, C. Y. Tay et al., "Comparative analysis of the full genome of Helicobacter pylori isolate Sahul64 identifies genes of high divergence," Journal of Bacteriology, vol. 196, pp. 1073-1083, 2014.

[41] K. Tamura, M. Nei, and S. Kumar, "Prospects for inferring very large phylogenies by using the neighbor-joining method," Proceedings of the National Academy of Sciences of the United States of America, vol. 101, no. 30, pp. 11030-11035, 2004.

[42] S. Guindon, J. Dufayard, V. Lefort, M. Anisimova, W. Hordijk, and O. Gascuel, "New algorithms and methods to estimate maximum-likelihood phylogenies: assessing the performance of PhyML 3.0," Systematic Biology, vol. 59, no. 3, pp. 307-321, 2010.

[43] M. L. Acencio and N. Lemke, "Towards the prediction of essential genes by integration of network topology, cellular localization and biological process information," BMC Bioinformatics, vol. 10, article 1471, p. 290, 2009.

[44] E. Giombini, M. Orsini, D. Carrabino, and A. Tramontano, "An automatic method for identifying surface proteins in bacteria: SLEP," BMC Bioinformatics, vol. 11, article 39, 2010.

[45] C. Yu, C. Lin, and J. Hwang, "Predicting subcellular localization of proteins for Gram-negative bacteria by support vector machines based on n-peptide compositions," Protein Science, vol. 13, no. 5, pp. 1402-1406, 2004.

[46] J. L. Gardy, M. R. Laird, F. Chen et al., "PSORTb v.2.0: expanded prediction of bacterial protein subcellular localization and insights gained from comparative proteome analysis," Bioinformatics, vol. 21, no. 5, pp. 617-623, 2005.

[47] L. Du and Y. Luo, "Structure of a hexameric form of RadA recombinase from Methanococcus voltae," Acta Crystallographica Section F Structural Biology and Crystallization Communications, vol. 68, no. 5, pp. 511-516, 2012.

[48] R. Morita, S. Nakane, A. Shimada et al., "Molecular mechanisms of the whole DNA repair system: a comparison of bacterial and eukaryotic systems," Journal of Nucleic Acids, vol. 2010, Article ID 179594, 32 pages, 2010.

[49] O. Moldavski, O. Levin-Kravets, T. Ziv, Z. Adam, and G. Prag, "The hetero-hexameric nature of a chloroplast AAA+ FtsH protease contributes to its thermodynamic stability," PLOS ONE, vol. 7, no. 4, Article ID e36008, 2012.

[50] D. Beier, G. Spohn, R. Rappuoli, and V. Scarlato, "Identification and characterization of an operon of Helicobacter pylori that is involved in motility and stress adaptation," Journal of Bacteriology, vol. 179, no. 15, pp. 4676-4683, 1997.

[51] M. Kiran, A. Chauhan, R. Dziedzic et al., "Mycobacterium tuberculosis $\mathrm{ftsH}$ expression in response to stress and viability," Tuberculosis, vol. 89, supplement 1, pp. S70-S73, 2009.

[52] T. L. Cover, P. I. Hanson, and J. E. Heuser, "Acid-induced dissociation of VacA, the Helicobacter pylori vacuolating cytotoxin, reveals its pattern of assembly," Journal of Cell Biology, vol. 138, no. 4, pp. 759-769, 1997.

[53] D. Kuck, B. Kolmerer, C. Iking-Konert, P. H. Krammer, W. Stremmel, and J. Rudi, "Vacuolating cytotoxin of Helicobacter 
pylori induces apoptosis in the human gastric epithelial cell line AGS," Infection and Immunity, vol. 69, no. 8, pp. 5080-5087, 2001.

[54] S. L. Palframan, T. Kwok, and K. Gabriel, "Vacuolating cytotoxin A (VacA), a key toxin for Helicobacter pylori pathogenesis," Frontiers in Cellular and Infection Microbiology, vol. 2, article 92, 2012.

[55] P. Mossey, A. Hudacek, and A. Das, "Agrobacterium tumefaciens type IV secretion protein VirB3 is an inner membrane protein and requires VirB4, VirB7, and VirB8 for stabilization," Journal of Bacteriology, vol. 192, no. 11, pp. 2830-2838, 2010.

[56] K. Walldén, R. Williams, J. Yan et al., "Structure of the VirB4 ATPase, alone and bound to the core complex of a type IV secretion system," Proceedings of the National Academy of Sciences of the United States of America, vol. 109, no. 28, pp. 11348-11353, 2012. 


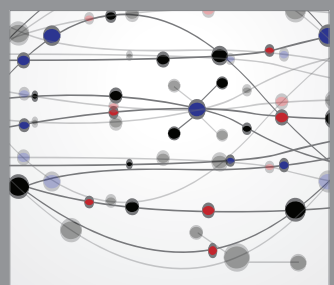

The Scientific World Journal
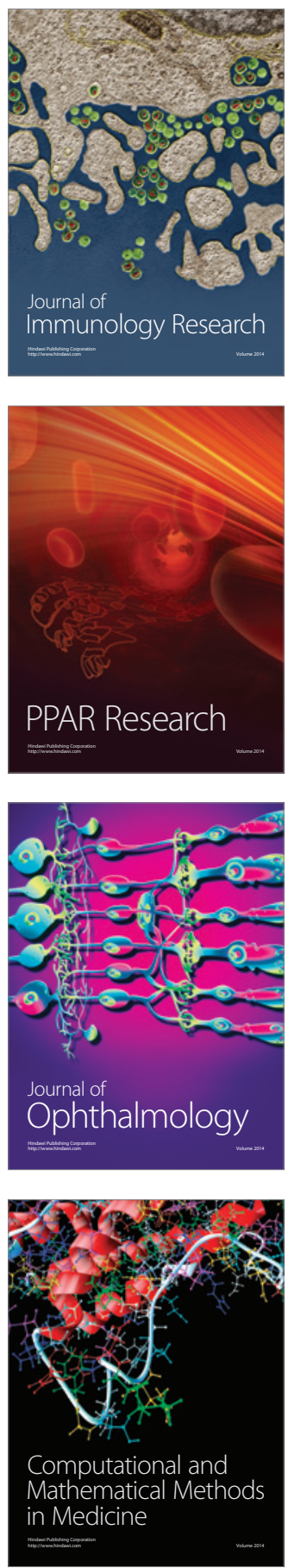

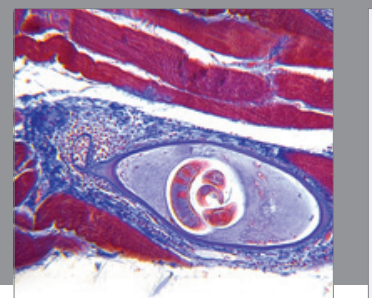

Gastroenterology

Research and Practice
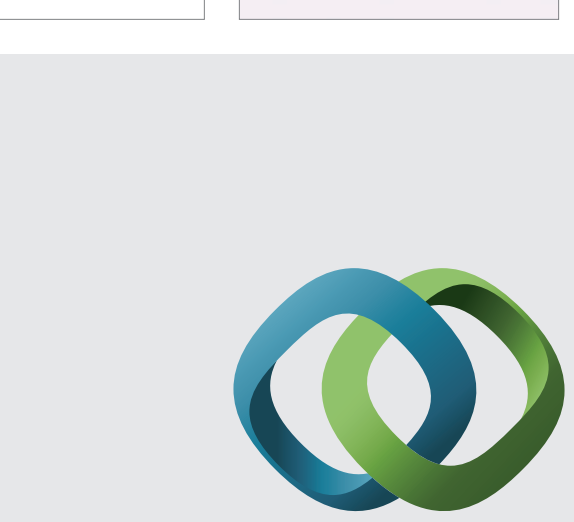

\section{Hindawi}

Submit your manuscripts at

http://www.hindawi.com
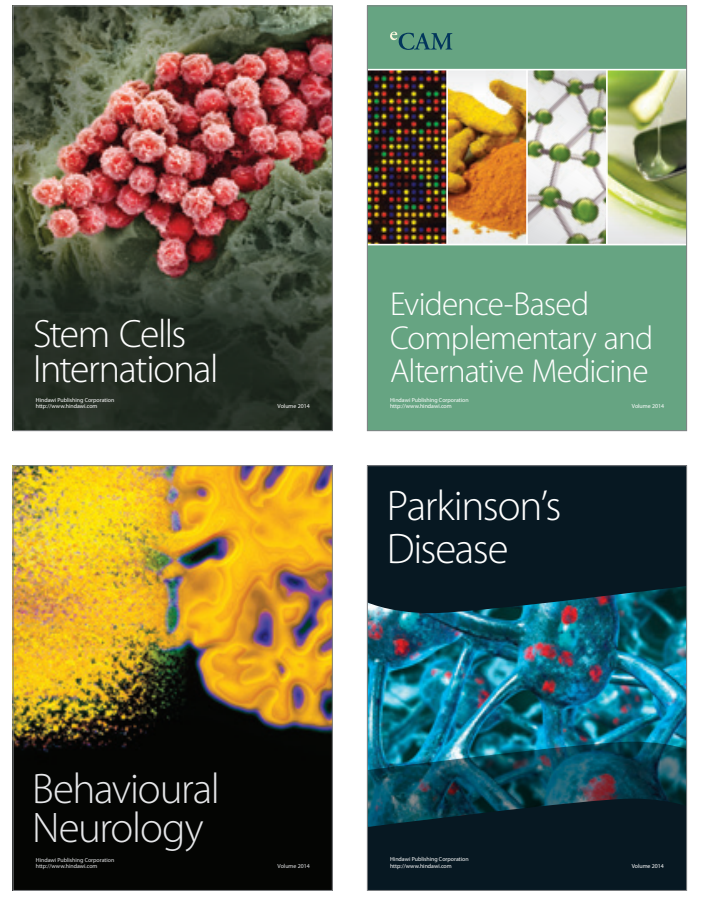
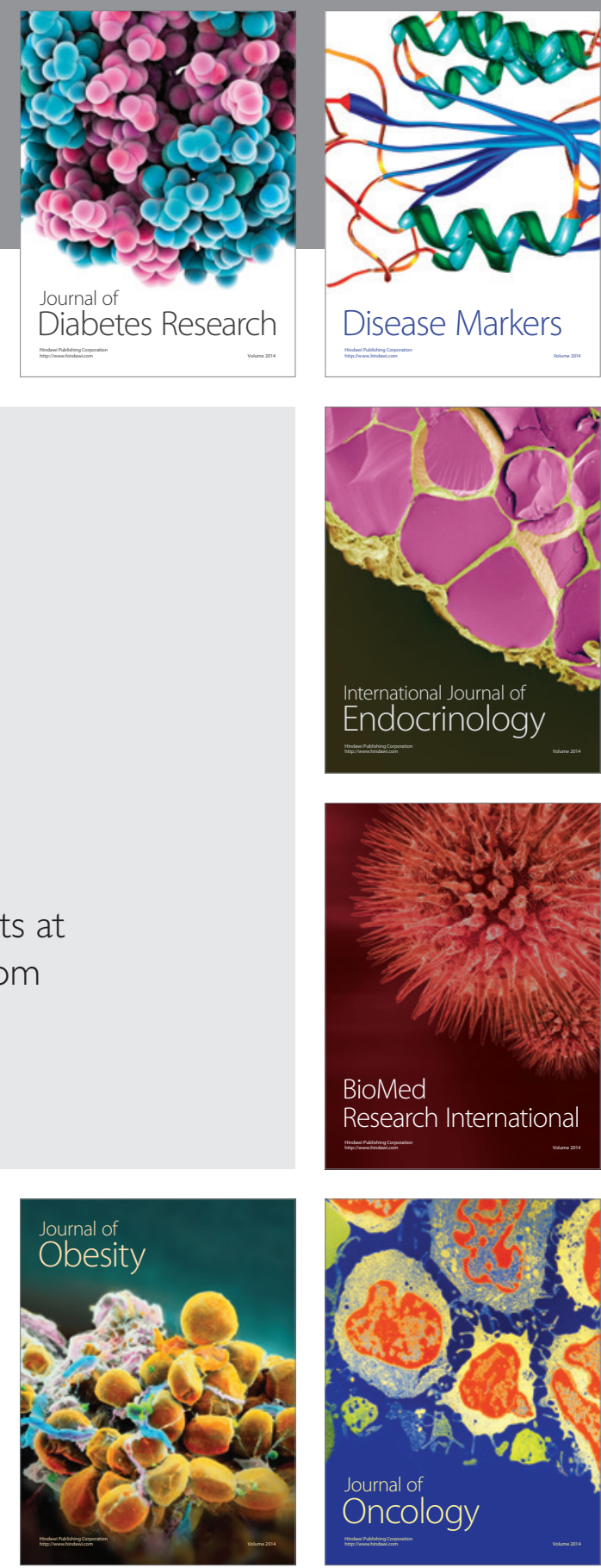

Disease Markers
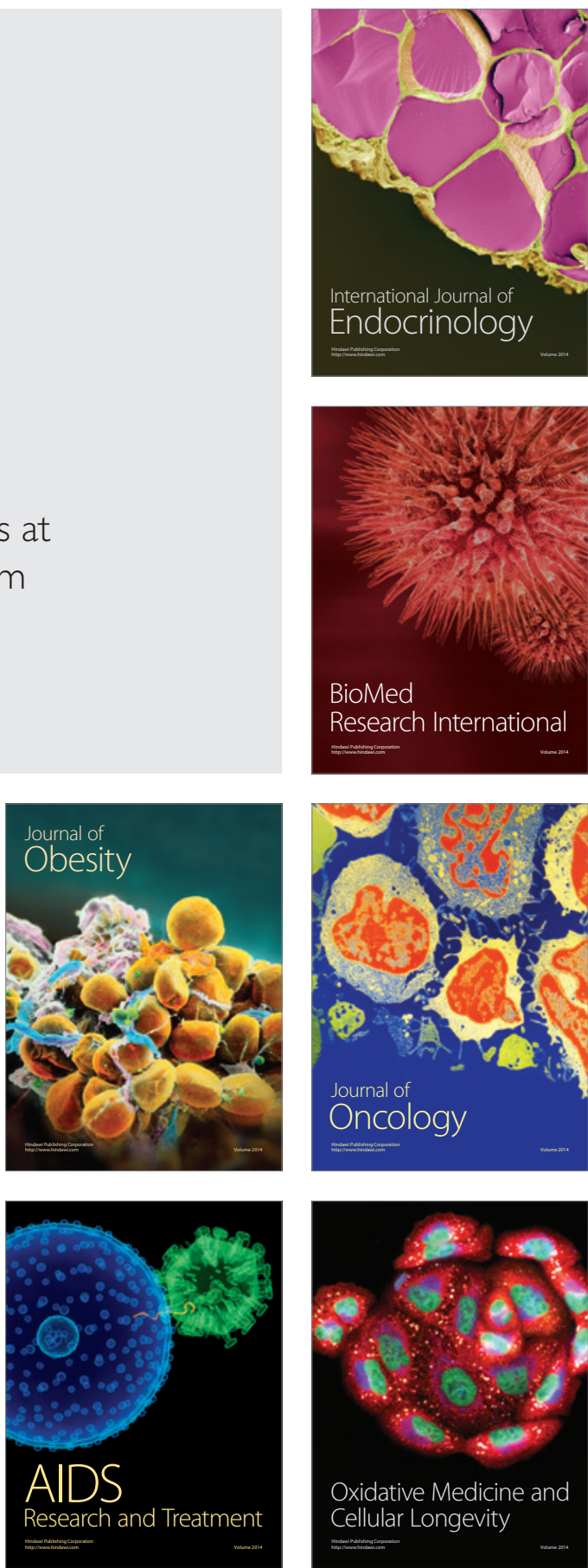